\title{
Topological quantum critical point in triple-Weyl semimetal: non-Fermi-liquid behaviors and instabilities
}

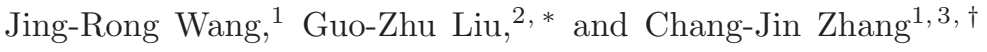 \\ ${ }^{1}$ Anhui Province Key Laboratory of Condensed Matter Physics at Extreme Conditions, \\ High Magnetic Field Laboratory of the Chinese Academy of Sciences, Hefei, Anhui 230031, China \\ ${ }^{2}$ Department of Modern Physics, University of Science and Technology of China, Hefei, Anhui 230026, China \\ ${ }^{3}$ Institute of Physical Science and Information Technology, Anhui University, Hefei, Anhui 230601, China
}

\begin{abstract}
We study the quantum critical phenomena emerging at the transition from triple-Weyl semimetal to band insulator, which is a topological phase transition described by the change of topological invariant. The critical point realizes a new type of semimetal state in which the fermion dispersion is cubic along two directions and quadratic along the third. Our renormalization group analysis reveals that, the Coulomb interaction is marginal at low energies and even arbitrarily weak Coulomb interaction suffices to induce an infrared fixed point. We compute a number of observable quantities, and show that they all exhibit non-Fermi liquid behaviors at the fixed point. When the interplay between the Coulomb and short-range four-fermion interactions is considered, the system becomes unstable below a finite energy scale. The system undergoes a first-order topological transition when the fermion flavor $N$ is small, and enters into a nematic phase if $N$ is large enough. Non-Fermi liquid behaviors are hidden by the instability at low temperatures, but can still be observed at higher temperatures. Experimental detection of the predicted phenomena is discussed.
\end{abstract}

\section{INTRODUCTION}

Weakly interacting fermion systems are well described by Fermi liquid (FL) theory [1, 2]. Inter-particle interactions induce a variety of continuous phase transitions, such as magnetically ordered transition [3, 4], chargedensity-wave transition [5], and nematic transition [6]. This type of transition happens between two phases with distinct symmetries. According to Ginzburg-LandauWilson (GLW) theory [3], one can define a local order parameter to describe such a transition. At the quantum critical point (QCP), fermionic excitations interact strongly with the quantum fluctuation of order parameter, which often leads to breakdown of FL theory [3 7 ]. However, not all phase transitions are classified by the change of symmetries. Transitions may occur between phases that respect the same symmetries but are topologically distinct [8, 9]. These transitions are beyond the paradigm of GLW theory, and could be described by the change of global topological invariant.

In recent years, a plethora of semimetal (SM) materials have been discovered and extensively investigated 10 20]. Under suitable conditions, SM states emerge at the toplogical QCP (TQCP) between two topologically distinct phases [21 33]. For instance, three-dimensional (3D) topological insulator (TI) can be turned into a band insulator (BI), with gapless 3D Dirac SM (DSM) state emerging at TI-BI QCP [21, 23, 34, 35].

Inter-particle interaction effect at TQCP is a nontrivial issue and has attracted particular interest recently 23 26, 31]. The fermions do not couple to any order parameter at the TQCP. Their properties are determined

\footnotetext{
*Corresponding author: gzliu@ustc.edu.cn

${ }^{\dagger}$ Corresponding author: zhangcj@hmfl.ac.cn
}

mainly by the Coulomb interaction, which is long-ranged because the density of states (DOS) vanishes at Fermi level. The effects of Coulomb interaction are diverse in various SM systems, depending sensitively on the spatial dimensionality and the fermion energy dispersion [23. 26, 31, 36 44.

In this article, we consider the transition between topological triple-Weyl SM (WSM) and BI. In a triple-WSM, the fermion energy spectrum disperses cubically along two directions and linearly along the third one, and the monopole charges of one pair of Weyl points are \pm 3 [42] 54. In the process of turning triple-WSM into BI, two Weyl points carrying opposite monopole charges merge to form one single band-touching point that carries zero monopole charge. This is a topological quantum phase transition (TQPT) since the monopole charge is finite in one phase and zero in the other. The merging process is shown in Fig. 1] At the TQCP, the fermion dispersion is cubic along two directions and quadratic along the third, which defines a new gapless SM state.

We investigate the influence of long-range Coulomb interaction on the triple-WSM-BI TQCP by carrying out an extensive renormalization group (RG) analysis. The Coulomb interaction is found to be marginal in the lowenergy regime. The system flows to an infrared fixed point, at which the interaction strength takes a finite value. We compute a number of observable quantities and demonstrate that they all exhibit striking non-FL (NFL) behaviors at such a fixed point.

We then study the possible instabilities of this fixed point by analyzing the interplay between the Coulomb interaction and some sorts of short-range four-fermion interactions. Our finding is that, the system undergoes a first order TQPT when the fermion flavor $N$ is small and develops a nematic order if $N$ is sufficiently large. The NFL behaviors are hidden at low temperatures due to the instability, but still have observable effects at higher tem- 
(a)

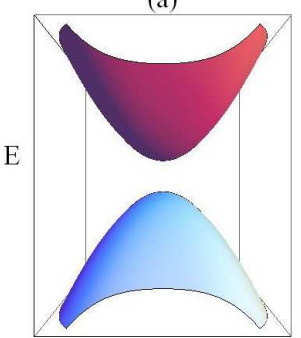

$k_{z}$ (b)

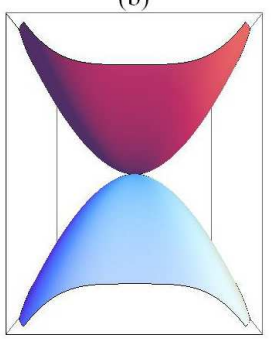

$k_{z}$ (c)

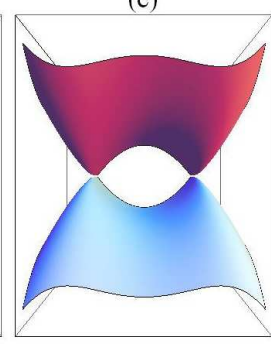

$k_{z}$
FIG. 1: As a triple-WSM is converted into a normal BI, two band-touching points merge to one single point at the TQCP. The fermion dispersion can be universally expressed in the form $E= \pm \sqrt{B^{2} k_{\perp}^{6}+\left(A k_{z}^{2}-\Delta\right)^{2}}$. (a) BI with $\Delta<0$; (b) TQCP with $\Delta=0$; (c) Triple-WSM with $\Delta>0$.

peratures. All these results, summarized in the schematic phase diagram given by Fig. 2, can be verified by experiments.

The rest of the paper will be organized as follows. In Sec. III, the effective low-energy action of the TQCP is introduced and described. In Sec. III, the coupled RG equations for model parameters are presented, and the RG solutions are analyzed. The NFL behaviors of observable quantities are discussed in Sec. IV] The possible phase-transition instabilities induced by the Coulomb and short-range interactions are investigated in Sec. V] In Sec. VI, the experimental detection of our theoretical predictions is briefly discussed. All of calculational details are presented in the Appendices.

\section{THE MODEL}

At the triple-WSM-BI TQCP, the free Hamiltonian of the gapless fermions takes the form

$$
H_{f}=\sum_{i=1}^{3} \int d^{3} \mathbf{x} \psi_{a}^{\dagger}(\mathbf{x}) d_{i}(\mathbf{x}) \sigma_{i} \psi_{a}(\mathbf{x})
$$

where $d_{1}(\mathbf{x})=B i\left(\partial_{x}^{3}-3 \partial_{x} \partial_{y}^{2}\right), d_{2}(\mathbf{x})=B i\left(\partial_{y}^{3}-3 \partial_{y} \partial_{x}^{2}\right)$, and $d_{3}(\mathbf{x})=-A \partial_{z}^{2}$. The field operator $\psi_{a}$ is a twocomponent spinor, and repeated indices of $a$ implies the summation from 1 to $N$, where $N$ is the fermion flavor. $\sigma_{1,2,3}$ are the Pauli matrices, and $B$ and $A$ are model parameters. The fermion energy dispersion is $E= \pm \sqrt{B^{2} k_{\perp}^{6}+A^{2} k_{z}^{4}}$, where $k_{\perp}^{2}=k_{x}^{2}+k_{y}^{2}$. The Coulomb interaction can be described by

$$
H_{C}=\frac{1}{4 \pi} \int d^{3} \mathbf{x} d^{3} \mathbf{x}^{\prime} \rho_{a}(\mathbf{x}) \frac{e^{2}}{\epsilon\left|\mathbf{x}-\mathbf{x}^{\prime}\right|} \rho_{a}\left(\mathbf{x}^{\prime}\right),
$$

where $\rho_{a}(\mathbf{x})=\psi_{a}^{\dagger}(\mathbf{x}) \psi_{a}(\mathbf{x})$ is fermion density operator, $e$ electric charge, and $\epsilon$ dielectric constant. One can treat Coulomb interaction by defining an auxiliary boson field

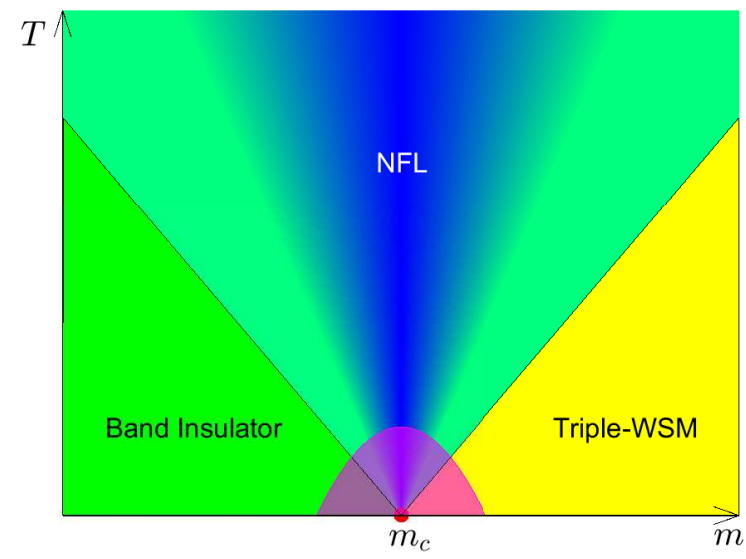

FIG. 2: Phase diagram on $m-T$ plane, where $m$ tunes the transition and $T$ is temperature. The TQCP $\left(m_{c}\right)$ is broadened into a NFL-like quantum critical regime at finite $T$. Magenta dome appearing at low $T$ represents either the formation of nematic order or the occurrence of first-order TQPT.

$\phi$ via the Hubbard-Stratonovich transformation 23 25, 31, 36 43.

The total effective action is formally expressed as $S=$ $S_{\psi}+S_{\phi}+S_{\psi \phi}$, where

$$
\begin{aligned}
S_{\psi} & =\int \frac{d \omega}{2 \pi} \frac{d^{3} \mathbf{k}}{(2 \pi)^{3}} \psi_{a}^{\dagger}\left(i \omega-\mathcal{H}_{f}(\mathbf{k})\right) \psi_{a} \\
S_{\phi} & =\int \frac{d \omega}{2 \pi} \frac{d^{3} \mathbf{k}}{(2 \pi)^{3}} \phi\left(k_{\perp}^{2}+\eta k_{z}^{2}\right) \phi \\
S_{\psi \phi} & =i g \int d \tau d^{3} \mathbf{x} \phi \psi_{a}^{\dagger} \psi_{a} .
\end{aligned}
$$

Here, $g=\sqrt{4 \pi} e / \sqrt{\epsilon}$. Because the fermion dispersion is anisotropic, the three momentum components need to be re-scaled differently. To facilitate RG calculations, we have introduced a parameter $\eta$. The bare fermion propagator is given by

$$
G_{0}(i \omega, \mathbf{k})=\frac{1}{i \omega-d_{1}(\mathbf{k}) \sigma_{1}-d_{2}(\mathbf{k}) \sigma_{2}-d_{3}(\mathbf{k}) \sigma_{3}},
$$

where $d_{1}(\mathbf{k})=B\left(k_{x}^{3}-3 k_{x} k_{y}^{2}\right), d_{2}(\mathbf{k})=B\left(k_{y}^{3}-3 k_{y} k_{x}^{2}\right)$, and $d_{3}(\mathbf{k})=A k_{z}^{2}$. The bare boson propagator reads

$$
D_{0}(\mathbf{q})=\frac{1}{q_{\perp}^{2}+\eta q_{z}^{2}},
$$

which is clearly long-ranged.

\section{RENORMALIZATION GROUP ANALYSIS}

We now employ the RG method [55] to study the Coulomb interaction on the low-energy properties of fermions. The momentum shell to be integrated out is chosen as $b \Lambda<\sqrt{B^{2} k_{\perp}^{6}+A^{2} k_{z}^{4}}<\Lambda$, where $\Lambda$ is some 
high-energy cutoff and $b=e^{-\ell}$ with $\ell$ being the flow parameter. After performing tedious calculations, we obtain the fermion self-energy

$$
\Sigma(i \omega, \mathbf{k}) \approx-\left[d_{1}(\mathbf{k}) \sigma_{1}+d_{2}(\mathbf{k}) \sigma_{2}\right] C_{2} \ell-A k_{z}^{2} \sigma_{3} C_{3} \ell .
$$

The expressions of $C_{2}$ and $C_{3}$ are complicated and can be found in Appendix $\mathrm{A}$ According to Appendix B, the boson self-energy calculated in the static limit is given by

$$
\Pi(\mathbf{q}) \approx q_{\perp}^{2} C_{\perp} \ell+q_{z}^{2} C_{z} \ell
$$

where

$$
\begin{aligned}
C_{\perp} & =\frac{9 g^{2}}{40 \pi^{2} \sqrt{A \Lambda}}, \\
C_{z} & =\frac{\Gamma\left(\frac{3}{4}\right) \Gamma\left(\frac{4}{3}\right) g^{2} \sqrt{A}}{24 \pi^{2} \Gamma\left(\frac{25}{12}\right) B^{2 / 3} \Lambda^{5 / 6}} .
\end{aligned}
$$

According to the calculations presented in Appendix C the coupled RG equations for model parameters are

$$
\begin{aligned}
& \frac{d B}{d \ell}=C_{2} B \\
& \frac{d A}{d \ell}=C_{3} A \\
& \frac{d \alpha}{d \ell}=\left(\frac{1}{2}-N C_{\perp}-\frac{C_{3}}{2}\right) \alpha \\
& \frac{d \beta}{d \ell}=\left(\frac{5}{6}+\frac{1}{2} C_{3}-\frac{2}{3} C_{2}-N \beta\right) \beta \\
& \frac{d \eta}{d \ell}=\left(-\frac{1}{3}-N C_{\perp}+N \beta\right) \eta \\
& \frac{d \zeta}{d \ell}=\left(-\frac{1}{3}+\frac{2}{3} C_{2}-C_{3}-N C_{\perp}+N \beta\right) \zeta .
\end{aligned}
$$

Here, we define three new parameters: $\alpha=5 C_{\perp} / 9 \pi$, $\beta=C_{z} / \eta$, and $\zeta=\eta B^{2 / 3} \Lambda^{1 / 3} / A$. The parameter $\alpha$ represents the effective Coulomb interaction strength, whereas the parameter $\beta$ is related to the dynamical screening of Coulomb interaction. In our RG calculations, $1 / N$ serves as a formal control parameter.

We plot the flow diagrams on the $\alpha-\beta$ plane in Figs. 3(a) and 3(b) based on the solutions of Eqs. (12)(17). There is a stable infrared fixed point $\left(\alpha^{*}, \beta^{*}\right)$. In the lowest energy limit, $C_{2}, C_{3}$, and $C_{\perp}$ approach to three constants $C_{2}^{*}, C_{3}^{*}$, and $C_{\perp}^{*}$, respectively. The values of the constants $\alpha^{*}, \beta^{*}, C_{2}^{*}, C_{3}^{*}$, and $C_{\perp}^{*}$ are shown in Fig. 3(c), and can be found in Appendix [C. The constant $C_{3}^{*}<0$, but other constants are positive. At low energies, the parameters $B$ and $A$ behave as

$$
\begin{aligned}
& B \sim e^{C_{2}^{*} \ell}, \\
& A \sim e^{C_{3}^{*} \ell} .
\end{aligned}
$$

As the energy decreases, $B$ increases indefinitely, while $A$ goes to zero quickly. Since $B$ and $A$ enter into almost
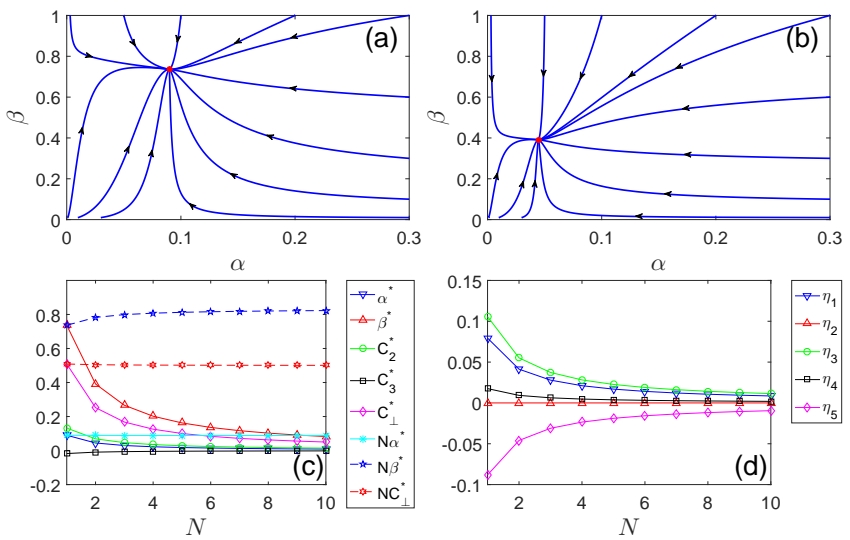

FIG. 3: Flow diagram on $\alpha-\beta$ plane for $N=1$ is given in (a) and for $N=2$ in (b). Red point $\left(\alpha^{*}, \beta^{*}\right)$ is an infrared fixed point. (c) and (d) show the $N$-dependence of some parameters.

all the observable quantities, such a renormalization will certainly have observable effects.

Including polarization $\Pi(\mathbf{q})$ into the boson propagator leads to renormalized Coulomb interaction

$$
D(\mathbf{q})=\frac{1}{D_{0}^{-1}(\mathbf{q})+\Pi(\mathbf{q})} \sim \frac{1}{q_{\perp}^{2-3 N C_{\perp}^{*}}+q_{z}^{2-2 N \beta^{*}}} .
$$

Since $C_{\perp}^{*}$ and $\beta^{*}$ are both positive, the Coulomb interaction is substantially screened.

\section{OBSERVABLE QUANTITIES}

The infrared fixed point $\left(\alpha^{*}, \beta^{*}\right)$ is characterized by the emergence of striking NFL behaviors. To demonstrate this, we will compute a number of observable quantities, including the fermion DOS, specific heat, compressibility, dynamical conductivities, and diamagnetic susceptibilities. The detailed calculations of observable quantities can be found in Appendices $\mathrm{D}$ and $\mathrm{E}$

We first consider the non-interacting limit. It is easy to get the fermion DOS

$$
\rho(\omega)=\frac{c_{1}}{\sqrt{A} B^{2 / 3}} \omega^{1 / 6} .
$$

Apparently, $\rho(\omega)$ vanishes in the limit $\omega \rightarrow 0$, which is a common feature of most SMs and renders that the Coulomb interaction is long-ranged. The specific heat $C_{v}(T)$ and compressibility $\kappa(T)$ are

$$
\begin{aligned}
C_{v}(T) & =\frac{c_{2}}{\sqrt{A} B^{2 / 3}} T^{7 / 6}, \\
\kappa(T) & =\frac{c_{3}}{\sqrt{A} B^{2 / 3}} T^{1 / 6},
\end{aligned}
$$

respectively. The dynamical conductivity within $x-y$ 
TABLE I: The energy or temperature dependence of observable quantities at TQCP between triple-WSM and BI. The interaction-induced extra exponents are shown in Fig. 3(d) and also in Appendix E

\begin{tabular}{|c|c|c|c|c|c|c|c|}
\hline \hline & $\rho(\omega)$ & $C_{v}(T)$ & $\kappa(T)$ & $\sigma_{\perp \perp}(\omega)$ & $\sigma_{z z}(\omega)$ & $\chi_{D}^{\perp}(T)$ & $\chi_{D}^{z}(T)$ \\
\hline Free & $\omega^{1 / 6}$ & $T^{7 / 6}$ & $T^{1 / 6}$ & $\omega^{1 / 2}$ & $\omega^{1 / 6}$ & $T^{1 / 2}$ & $T^{5 / 6}$ \\
\hline Interacting & $\omega^{1 / 6+\eta_{1}}$ & $T^{7 / 6+\eta_{1}}$ & $T^{1 / 6+\eta_{1}}$ & $\omega^{1 / 2+\eta_{2}}$ & $\omega^{1 / 6+\eta_{3}}$ & $T^{1 / 2+\eta_{4}}$ & $T^{5 / 6+\eta_{5}}$ \\
\hline \hline
\end{tabular}

plane, $\sigma_{\perp \perp}(\omega)$, and the one along $z$-axis, $\sigma_{z z}(\omega)$, are

$$
\begin{aligned}
\sigma_{\perp \perp}(\omega) & =\frac{c_{4} e^{2}}{\sqrt{A}} \omega^{1 / 2}, \\
\sigma_{z z}(\omega) & =\frac{c_{5} \sqrt{A} e^{2}}{B^{2 / 3}} \omega^{1 / 6} .
\end{aligned}
$$

For the external fields applied within the $x-y$ plane and along the $z$-axis, the diamagnetic susceptibilities are respectively given by

$$
\begin{aligned}
& \Delta \chi_{D}^{\perp}(T)=c_{6} \sqrt{A} e^{2} T^{1 / 2}, \\
& \Delta \chi_{D}^{z}(T)=c_{7} \frac{B^{2 / 3} e^{2}}{\sqrt{A}} T^{5 / 6},
\end{aligned}
$$

where $\Delta \chi_{D}^{\frac{1}{D}}(T)=\chi_{D}^{\frac{1}{D}}(T)-\chi_{D}^{\frac{1}{D} 0}$ and $\Delta \chi_{D}^{z}(T)=\chi_{D}^{z}(T)-$ $\chi_{D 0}^{z}$, with $\chi_{D 0}^{\perp}$ and $\chi_{D 0}^{z}$ being the residual values of the $T \rightarrow 0$ limit. The values of constants $c_{1}, c_{2}, \ldots, c_{7}$ are given in Appendix D

We then incorporate the corrections induced by the Coulomb interaction. Making use of the RG results of $B(\ell)$ and $A(\ell)$, and then employing the scaling relation $\omega=\omega_{0} e^{-\ell}$ or $T=T_{0} e^{-\ell}$, we can obtain the renormalized observable quantities. First of all, the DOS becomes

$$
\rho(\omega) \sim \omega^{1 / 6+\eta_{1}},
$$

where $\eta_{1}>0$. Comparing Eq. (21) to Eq. (28), we find that Coulomb interaction effectively suppresses fermion DOS. Interaction corrections modify specific heat and compressibility to

$$
\begin{aligned}
C_{v}(T) & \sim T^{7 / 6+\eta_{1}}, \\
\kappa(T) & \sim T^{1 / 6+\eta_{1}} .
\end{aligned}
$$

These two quantities are also suppressed. The extra exponent $\eta_{1}$ appearing in $\rho(\omega), C_{v}(T)$, and $\kappa(T)$ is exactly the same, which reflects the fact that $\rho(\omega), C_{v}(T)$, and $\kappa(T)$ display the same dependence on $B$ and $A$, as shown in Eqs. (21), (22), and (23).

According to Eqs. (24)- (27), the charge $e$ enters into the expressions of dynamical conductivities and diamagnetic susceptibilities. The RG flow of $e$ naturally affects the low-energy properties of these quantities. From the $\ell$-dependence of $B, A$, and $e$, we find that

$$
\begin{aligned}
\sigma_{\perp \perp}(\omega) & \sim \omega^{1 / 2+\eta_{2}}, \\
\sigma_{z z}(\omega) & \sim \omega^{1 / 6+\eta_{3}},
\end{aligned}
$$

where $\eta_{2}=0$ and $\eta_{3}$ is a positive constant. Notice that the $\omega$-dependence of $\sigma_{\perp \perp}$ is unchanged. The reason is that, $e^{2}$ and $\sqrt{A}$ are renormalized in precisely the same way by Coulomb interaction, which ensures that $\sigma_{\perp \perp} \propto$ $e^{2} / \sqrt{A}$ remains intact. Eq. (25) and Eq. (32) indicate that $\sigma_{z z}$ is suppressed by Coulomb interaction.

After including the interaction corrections, the diamagnetic susceptibilities become

$$
\begin{aligned}
\Delta \chi_{D}^{\perp}(T) & \sim T^{1 / 2+\eta_{4}}, \\
\Delta \chi_{D}^{z}(T) & \sim T^{5 / 6+\eta_{5}},
\end{aligned}
$$

where $\eta_{4}>0$ but $\eta_{5}<0$.

We summarize the results for observable quantities in Table I. The power-law corrections indicate that Coulomb interaction induces typical NFL behaviors.

\section{INSTABILITY DRIVEN BY COULOMB INTERACTION}

An interesting consequence of the Coulomb interaction is to dynamically generate some types of short-range four-fermion coupling [37, 38, [56, 57]. If the four-fermion interaction flows to strong coupling regime, the system would become unstable. Motivated by previous works on Luttinger SM 37, 38], we now study the possible instabilities driven by Coulomb interaction through analyzing the interplay of Coulomb and four-fermion interactions at the TQCP under consideration.

There are four types of four-fermion interactions: $\lambda_{1}\left(\psi^{\dagger} \sigma_{1} \psi\right)^{2}, \lambda_{2}\left(\psi^{\dagger} \sigma_{2} \psi\right)^{2}, \lambda_{3}\left(\psi^{\dagger} \sigma_{3} \psi\right)^{2}$, and $\lambda_{0}\left(\psi^{\dagger} \psi\right)^{2}$. After decoupling each four-fermion coupling term, one obtains a fermion bilinear. Each fermion bilinear has its own meaning. Below, we study these four cases separately in order.

We first consider the following coupling

$$
S_{\psi^{4}}=\frac{1}{N} \int d \tau d^{3} \mathbf{x} \lambda_{1}\left(\psi_{a}^{\dagger} \sigma_{1} \psi_{a}\right)^{2} .
$$

According to the RG analysis shown in Appendix $\mathbb{E}$ its coupling parameter $\lambda_{1}$ satisfies the flow equation

$$
\frac{d \lambda_{1}}{d \ell}=-\frac{1}{6} \lambda_{1}+\left(1-\frac{2}{5 N}\right) \lambda_{1}^{2}+F_{A} \lambda_{1}+F_{B},
$$

where the redefinition $\frac{5 \Gamma(5 / 4) \Gamma(4 / 3) \Lambda^{1 / 6}}{12 \pi^{2} \Gamma(19 / 12) \sqrt{A} B^{2 / 3}} \lambda_{1} \rightarrow \lambda_{1}$ has been employed. The factors $F_{A}$ and $F_{B}$ are induced 
by Coulomb interaction. The flows of $\lambda_{1}$ are presented in Fig. 4. We observe from Figs. 4(a) and 4(b) that, $\lambda_{1}(\ell)$ develops a finite value from zero as $\ell$ increases and $\lambda_{1}(\ell)$ diverges as $\ell \rightarrow \ell_{c}$ with $\ell_{c}$ being finite. The flow diagrams plotted in Figs. 4(c) and 4(d) show that this conclusion holds even when the initial value of $\alpha$ is arbitrarily small. As a result of the runaway flow of $\left(\psi^{\dagger} \sigma_{1} \psi\right)^{2}$, the corresponding fermion bilinear acquires a finite expectation value, namely $\Delta_{1} \equiv$ $\left\langle\psi^{\dagger} \sigma_{1} \psi\right\rangle \neq 0$. The original band-touching point is split into three different points: $\left(-\left(\Delta_{1} / B\right)^{1 / 3}, 0,0\right)$ and $\left(\frac{1}{2}\left(\Delta_{1} / B\right)^{1 / 3}, \pm \frac{\sqrt{3}}{2}\left(\Delta_{1} / B\right)^{1 / 3}, 0\right)$. One can identify $\Delta_{1}$ as a nematic order parameter, because the positions of these points break the equivalence between $x$ - and $y$-axis. Thus, the system enters into a nematic state. Around these three points, the fermion dispersion can be expressed as

$$
E= \pm \sqrt{9 B^{2 / 3} \Delta_{1}^{4 / 3} K_{\perp}^{2}+A^{2} K_{z}^{4}}
$$

which is linear along two directions and quadratic along the third. $\mathbf{K}$ is the momentum relative to the new touching points. Such a dispersion is the same as that of an anisotropic-WSM [29, 31], in which the Coulomb interaction is irrelevant at low energies, and the DOS, specific heat, and compressibility behave as $\rho(\omega) \sim \omega^{3 / 2}$, $C_{v}(T) \sim T^{5 / 2}$, and $\kappa(T) \sim T^{3 / 2}$, respectively. The mean value $\Delta_{1}$ is nonzero only at low $T$, and is destroyed as $T$ grows. Therefore, the NFL behaviors caused by Coulomb interaction are hidden at low $T$ by the nematic order, but re-appear at higher $T$ once the nematic order is destroyed by thermal fluctuation. The critical temperature $T_{n}$ can be roughly estimated by $\Lambda e^{-\ell_{c}}$.

Similar to $\left\langle\psi^{\dagger} \sigma_{1} \psi\right\rangle$, a nonzero $\Delta_{2} \equiv\left\langle\psi^{\dagger} \sigma_{2} \psi\right\rangle$ also splits the original band-touching point into three new points, which are located at $\left(0,-\left(\Delta_{2} / B\right)^{1 / 3}, 0\right)$ and $\left( \pm \frac{\sqrt{3}}{2}\left(\Delta_{2} / B\right)^{1 / 3}, \frac{1}{2}\left(\Delta_{2} / B\right)^{1 / 3}, 0\right)$. Thus, $\left\langle\psi^{\dagger} \sigma_{2} \psi\right\rangle$ also corresponds to nematic order parameter.

If $\Delta_{3} \equiv\left\langle\psi^{\dagger} \sigma_{3} \psi\right\rangle \neq 0$, the parameter $\Delta$ (defined in Fig. (1) becomes finite, i.e., $\Delta=-\Delta_{3}$, at the TQCP. Consequently, the transition between triple-WSM and BI becomes first-order [49], and the original TQCP is eliminated at low $T$. At higher $T$, thermal fluctuation forces $\Delta_{3}$ to vanish, and the effective fermion dispersion is still $E= \pm \sqrt{B^{2} k_{\perp}^{6}+A^{2} k_{z}^{4}}$. The Coulomb interaction leads to NFL behaviors at high $T$, which can be explored by measuring the observable quantities of Table [.

If $\Delta_{0} \equiv\left\langle\psi^{\dagger} \psi\right\rangle \neq 0$, the Fermi surface moves away from the band-touching point to a new level, since $\left\langle\psi^{\dagger} \psi\right\rangle$ plays the role of a finite chemical potential.

As demonstrated in Appendix E $\lambda_{2}$ and $\lambda_{3}$ are both generated from zero, and both diverge at some finite energy scale. However, $\lambda_{0}$ grows from zero and finally approaches to a constant in the lowest energy limit, which means that $\Delta_{0} \equiv 0$. Therefore, there are only two different instabilities, namely the formation of nematicity and
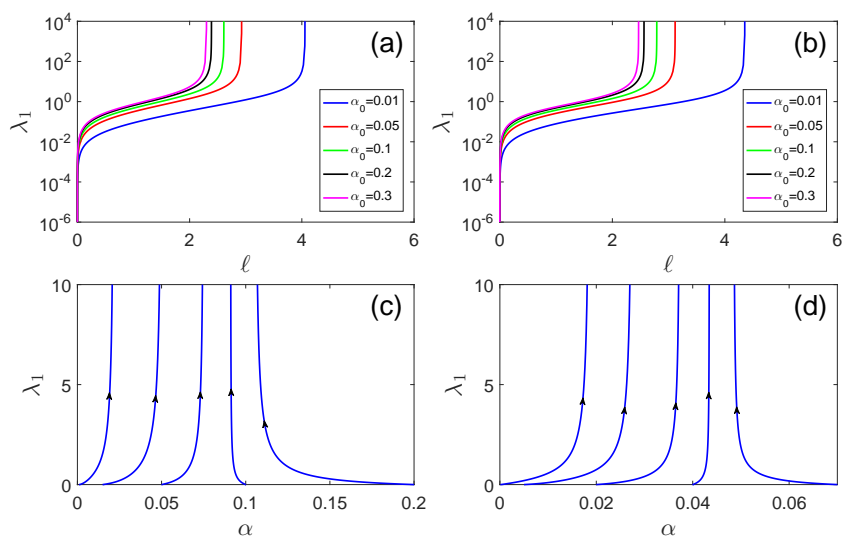

FIG. 4: Dependence of $\lambda_{1}$ on $\ell$ for $N=1$ is shown in (a) and for $N=2$ in (b). Flow diagram on $\alpha-\lambda_{1}$ plane for $N=1$ is shown in (c) and for $N=2$ in (d). Here, $\beta_{0}=0.1$.

the occurrence of first-order TQPT. The instability that would shift the Fermi level is excluded.

A natural question arises as to which out of the two instabilities is more favorable. As shown in Appendix $\mathrm{E}$. our numerical studies reveal that, within a wide range of the initial value of $\alpha, \lambda_{3}$ diverges most rapidly for $N=1,2$, whereas $\lambda_{1}$ and $\lambda_{2}$ diverge more quickly than $\lambda_{3}$ if $N \geq 3$. We thus conclude that, first-order TQPT occurs for $N=1,2$, but gapless nematic state is realized for $N \geq 3$.

\section{SUMMARY AND DISCUSSION}

In summary, we have investigated the quantum critical phenomena of the TQCP between the topological tripleWSM and the normal BI, and found that the long-range Coulomb interaction leads to a NFL infrared fixed point. This infrared fixed point can be experimentally probed by measuring the energy or temperature dependence of a number of observable quantities. We also have considered the interplay between Coulomb interaction and short-range interactions. Such an interplay can either causes a long-range nematic order or drive a first-order transition, depending on the value of the fermion flavor at TQCP. Comparing to some previously studied TQCPs 23 25, 31], this topological transition exhibits richer critical phenomena and provides interesting insight on the strong correlation effects at the topology-changing phase transitions.

Recent theoretical studies [30, 58, 59] predicted the presence of 3D cubic DSM state, in which the fermions have the same dispersion as triple-Weyl fermions. When a $3 \mathrm{D}$ cubic DSM is tuned to become a BI, the TQCP would exhibit the unusual properties predicted in this paper. It is proposed that $3 \mathrm{D}$ cubic-DSM can be realized in $\mathrm{Rb}(\mathrm{MoTe})_{3}$ and $\mathrm{Ti}(\mathrm{MoTe})_{3}$ [58], and $\mathrm{LiOsO}_{3}$ [59]. More candidate materials of triple-WSM or cubic-DSM might be discovered in the future. If such materials are pre- 
pared with high quality, one can tune them to approach the TQCP. We expect experiments would be performed to measure the observable quantities studied in this paper and to determine the nature of the low-energy instability. Quantum Monte Carlo simulations [60 63] may help address these issues.

Note Added - After we received the referees' reports, we had become aware of two preprints [64, 65], which study the influence of Coulomb interaction at the TQCP between double-WSM and BI.

\section{ACKNOWLEDGEMENTS}

We acknowledge the support from the National Key R\&D Program of China under Grants 2016YFA0300404 and 2017YFA0403600, and that from the National Natural Science Foundation of China under Grants 11574285, 11504379, 11674327, U1532267, and U1832209.

\section{Appendix A: Self-energy of Fermion}

The fermion self-energy is defined as

$$
\begin{aligned}
\Sigma(i \omega, \mathbf{k})= & g^{2} \int^{\prime} \frac{d \Omega}{2 \pi} \frac{d^{3} \mathbf{q}}{(2 \pi)^{3}} G_{0}(i \omega+i \Omega, \mathbf{k}+\mathbf{q}) \\
& \times D_{0}(i \Omega, \mathbf{q})
\end{aligned}
$$

where the free fermion propagator is

$$
G_{0}(i \omega, \mathbf{k})=\frac{1}{i \omega-\mathcal{H}_{f}(\mathbf{k})},
$$

where

$$
\mathcal{H}_{f}(\mathbf{k})=B\left[d_{1}(\mathbf{k}) \sigma_{1}+d_{2}(\mathbf{k}) \sigma_{2}\right]+A d_{3}(\mathbf{k}) \sigma_{3},
$$

with $d_{1}(\mathbf{k})=\left(k_{x}^{3}-3 k_{x} k_{y}^{2}\right), d_{2}(\mathbf{k})=\left(k_{y}^{3}-3 k_{y} k_{x}^{2}\right)$, and $d_{3}(\mathbf{k})=k_{z}^{2}$. The free boson propagator is given by

$$
D_{0}(i \Omega, \mathbf{q})=D_{0}(\mathbf{q})=\frac{1}{q_{x}^{2}+q_{y}^{2}+\eta q_{z}^{2}}=\frac{1}{q_{\perp}^{2}+\eta q_{z}^{2}} .
$$

Substituting Eqs. (A2) and (A4) into Eq. (A1), and retaining the leading order of contribution, we obtain

$$
\begin{aligned}
\Sigma(i \omega, \mathbf{k}) \approx & i \omega \Sigma_{1}-B\left[d_{1}(\mathbf{k}) \sigma_{1}+d_{2}(\mathbf{k}) \sigma_{2}\right] \Sigma_{2} \\
& -A d_{3}(\mathbf{k}) \sigma_{3} \Sigma_{3},
\end{aligned}
$$

where

$$
\begin{aligned}
\Sigma_{1}= & \frac{g^{2}}{4 \pi^{3}} \int^{\prime} d \Omega d q_{\perp} d\left|q_{z}\right| q_{\perp} \frac{\Omega^{2}-B^{2} q_{\perp}^{6}-A^{2} q_{z}^{4}}{\left(\Omega^{2}+B^{2} q_{\perp}^{6}+A^{2} q_{z}^{4}\right)^{2}} \\
& \times D_{0}(i \Omega, \mathbf{q}), \\
\Sigma_{2}= & \frac{g^{2}}{4 \pi^{3}} \int^{\prime} d \Omega d q_{\perp} d\left|q_{z}\right| q_{\perp}\left[\frac{\Omega^{2}-18 B^{2} q_{\perp}^{6}+A^{2} q_{z}^{4}}{\left(\Omega^{2}+B^{2} q_{\perp}^{6}+A^{2} q_{z}^{4}\right)^{2}}\right.
\end{aligned}
$$

$$
\begin{gathered}
+\frac{45 B^{4} q_{\perp}^{12}}{\left(\Omega^{2}+B^{2} q_{\perp}^{6}+A^{2} q_{z}^{4}\right)^{3}} \\
\left.-\frac{27 B^{6} q_{\perp}^{18}}{\left(\Omega^{2}+B^{2} q_{\perp}^{6}+A^{2} q_{z}^{4}\right)^{4}}\right] D_{0}(i \Omega, \mathbf{q}), \\
\Sigma_{3}= \\
+\frac{g^{2}}{4 \pi^{3}} \int^{\prime} d \Omega d q_{\perp} d\left|q_{z}\right| q_{\perp}\left[\frac{\Omega^{2}+B^{2} q_{\perp}^{6}-13 A^{2} q_{z}^{4}}{\left(\Omega^{2}+B^{2} q_{\perp}^{6}+A^{2} q_{z}^{4}\right)^{2}}\right. \\
\left.+\frac{16 A^{4} q_{z}^{8}}{\left(\Omega^{2}+B^{2} q_{\perp}^{6}+A^{2} q_{z}^{4}\right)^{3}}\right] D_{0}(i \Omega, \mathbf{q}) .
\end{gathered}
$$

In the following, we will employ the transformations

$$
E=\sqrt{B^{2} q_{\perp}^{6}+A^{2} q_{z}^{4}}, \quad \xi=\frac{B q_{\perp}^{3}}{A\left|q_{z}\right|^{2}},
$$

which are equivalent to

$$
q_{\perp}=\frac{\xi^{1 / 3} E^{1 / 3}}{B^{1 / 3}\left(1+\xi^{2}\right)^{1 / 6}}, \quad\left|q_{z}\right|=\frac{\sqrt{E}}{\sqrt{A}\left(1+\xi^{2}\right)^{1 / 4}} .
$$

The measures of the integrations satisfy the relation

$$
\begin{aligned}
d q_{\perp} d\left|q_{z}\right| & =\left\|\begin{array}{ll}
\frac{\partial q_{\perp}}{\partial E} & \frac{\partial q_{\perp}}{\partial \xi} \\
\frac{\partial\left|q_{z}\right|}{\partial E} & \frac{\partial\left|q_{z}\right|}{\partial \xi}
\end{array}\right\| d E d \xi \\
& =\left|\frac{\partial q_{\perp}}{\partial E} \frac{\partial\left|q_{z}\right|}{\partial \xi}-\frac{\partial q_{\perp}}{\partial \xi} \frac{\partial\left|q_{z}\right|}{\partial E}\right| d E d \xi \\
& =\frac{d E d \xi}{6 \sqrt{A} B^{1 / 3} E^{1 / 6} \xi^{2 / 3}\left(1+\xi^{2}\right)^{5 / 12}} .
\end{aligned}
$$

To proceed with RG calculations, we choose to integrate out high-energy modes defined in the momentum shell

$$
-\infty<\Omega<\infty, \quad b \Lambda<E<\Lambda,
$$

with $E=\sqrt{B^{2} q_{\perp}^{6}+A^{2} q_{z}^{4}}$ and $b=e^{-\ell}$. Utilizing the transformations Eqs. (A9)-A11), we obtain

$$
\begin{aligned}
\Sigma(i \omega, \mathbf{k}) \approx & -B\left[d_{1}(\mathbf{k}) \sigma_{1}+d_{2}(\mathbf{k}) \sigma_{2}\right] C_{2} \ell \\
& -A d_{3}(\mathbf{k}) \sigma_{3} C_{3} \ell,
\end{aligned}
$$

where

$$
\begin{aligned}
C_{2}= & \frac{g^{2}}{48 \pi^{2} \sqrt{A \Lambda}} \int_{0}^{+\infty} d \xi \frac{1}{\xi^{1 / 3}\left(1+\xi^{2}\right)^{13 / 12}} \\
& \times\left[2-17 \xi^{2}+\frac{135}{4} \frac{\xi^{4}}{\left(1+\xi^{2}\right)}-\frac{135}{8} \frac{\xi^{6}}{\left(1+\xi^{2}\right)^{2}}\right] \\
& \times \frac{1}{\xi^{2 / 3}\left(1+\xi^{2}\right)^{1 / 6}+\zeta}, \\
C_{3}= & \frac{g^{2}}{24 \pi^{2} \sqrt{A \Lambda}} \int_{0}^{+\infty} d \xi \frac{1}{\xi^{1 / 3}\left(1+\xi^{2}\right)^{13 / 12}} \\
& \times\left(\xi^{2}-6+\frac{6}{1+\xi^{2}}\right) \frac{1}{\xi^{2 / 3}\left(1+\xi^{2}\right)^{1 / 6}+\zeta},(\mathrm{A} 15) \\
\text { with } \zeta= & \frac{\eta B^{2 / 3} \Lambda^{1 / 3}}{A} .
\end{aligned}
$$




\section{Appendix B: Self-energy of Boson}

The boson self-energy is given by

$$
\begin{aligned}
\Pi(i \Omega, \mathbf{q})= & -N g^{2} \int \frac{d \omega}{2 \pi} \int^{\prime} \frac{d^{3} \mathbf{k}}{(2 \pi)^{3}} \operatorname{Tr}\left[G_{0}(i \omega, \mathbf{k})\right. \\
& \left.\times G_{0}(i \omega+i \Omega, \mathbf{k}+\mathbf{q})\right]
\end{aligned}
$$

Substituting Eq. (A2) into Eq. (B1), $\Pi(i \Omega, \mathbf{q})$ can be further written as

$$
\begin{aligned}
\Pi(i \Omega, \mathbf{q})= & 2 N g^{2} \int_{-\infty}^{+\infty} \frac{d \omega}{2 \pi} \int^{\prime} \frac{d^{3} \mathbf{k}}{(2 \pi)^{3}} \\
& \times \frac{1}{\left(\omega^{2}+E_{\mathbf{k}}^{2}\right)\left[(\omega+\Omega)^{2}+E_{\mathbf{k}+\mathbf{q}}^{2}\right]}\{\omega(\omega+\Omega) \\
& -B^{2}\left[d_{1}(\mathbf{k}) d_{1}(\mathbf{k}+\mathbf{q})+d_{2}(\mathbf{k}) d_{2}(\mathbf{k}+\mathbf{q})\right] \\
& \left.-A^{2} d_{3}(\mathbf{k}) d_{3}(\mathbf{k}+\mathbf{q})\right\},
\end{aligned}
$$

where $E_{\mathbf{k}}=\sqrt{B^{2} k_{\perp}^{6}+A^{2} k_{z}^{4}}$. Taking the static limit $\Omega=0$, and then expanding to the leading order of $q_{\perp}$ and $q_{z}$, we get

$$
\begin{aligned}
\Pi(0, \mathbf{q}) \approx & q_{\perp}^{2} N \frac{9 g^{2}}{16 \pi^{2}} \int^{\prime} d k_{\perp} d\left|k_{z}\right| k_{\perp}\left(\frac{2 B^{2} k_{\perp}^{4}}{E_{\mathbf{k}}^{3}}-\frac{B^{4} k_{\perp}^{10}}{E_{\mathbf{k}}^{5}}\right) \\
& +q_{z}^{2} N \frac{g^{2}}{2 \pi^{2}} \int^{\prime} d k_{\perp} d\left|k_{z}\right| k_{\perp} \frac{A^{2} k_{z}^{2} B^{2} k_{\perp}^{6}}{E_{\mathbf{k}}^{5}} .
\end{aligned}
$$

Using again the transformations Eqs. (A9)-A11), we finally obtain

$$
\Pi(0, \mathbf{q}) \approx q_{\perp}^{2} N C_{\perp} \ell+q_{z}^{2} N C_{z} \ell
$$

where

$$
C_{\perp}=\frac{9 g^{2}}{40 \pi^{2} \sqrt{A \Lambda}}, \quad C_{z}=\frac{\Gamma\left(\frac{3}{4}\right) \Gamma\left(\frac{4}{3}\right) g^{2} \sqrt{A}}{24 \pi^{2} \Gamma\left(\frac{25}{12}\right) B^{2 / 3} \Lambda^{5 / 6}} .
$$

\section{Appendix C: Derivation of RG equations}

The free action of fermion field $\psi$ is

$$
\begin{aligned}
S_{\psi}= & \int \frac{d \omega}{2 \pi} \frac{d^{3} \mathbf{k}}{(2 \pi)^{3}} \psi_{a}^{\dagger}(\omega, \mathbf{k})\left\{i \omega-B\left[d_{1}(\mathbf{k}) \sigma_{1}+d_{2}(\mathbf{k}) \sigma_{2}\right]\right. \\
& \left.-A d_{3}(\mathbf{k}) \sigma_{3}\right\} \psi_{a}(\omega, \mathbf{k}) .
\end{aligned}
$$

Upon incorporating the interaction corrections, we get

$$
\begin{aligned}
S_{\psi}= & \int \frac{d \omega}{2 \pi} \frac{d^{3} \mathbf{k}}{(2 \pi)^{3}} \psi_{a}^{\dagger}(\omega, \mathbf{k})\left\{i \omega-B\left[d_{1}(\mathbf{k}) \sigma_{1}+d_{2}(\mathbf{k}) \sigma_{2}\right]\right. \\
& \left.-A d_{3}(\mathbf{k}) \sigma_{3}+\Sigma(i \omega, \mathbf{k})\right\} \psi_{a}(\omega, \mathbf{k}) \\
\approx & \int \frac{d \omega}{2 \pi} \frac{d^{3} \mathbf{k}}{(2 \pi)^{3}} \psi_{a}^{\dagger}(\omega, \mathbf{k})\left\{i \omega-B\left[d_{1}(\mathbf{k}) \sigma_{1}+d_{2}(\mathbf{k}) \sigma_{2}\right]\right. \\
& \left.\times e^{C_{2} \ell}-A d_{3}(\mathbf{k}) \sigma_{3} e^{C_{3} \ell}\right\} \psi_{a}(\omega, \mathbf{k}) .
\end{aligned}
$$

Using the re-scaling transformations

$$
\begin{aligned}
\omega & =\omega^{\prime} e^{-\ell}, \\
k_{x} & =k_{x}^{\prime} e^{-\frac{\ell}{3}}, \\
k_{y} & =k_{y}^{\prime} e^{-\frac{\ell}{3}}, \\
k_{z} & =k_{z}^{\prime} e^{-\frac{\ell}{2}}, \\
\psi_{a} & =\psi_{a}^{\prime} e^{\frac{19}{12} \ell}, \\
B & =B^{\prime} e^{-C_{2} \ell}, \\
A & =A^{\prime} e^{-C_{3} \ell},
\end{aligned}
$$

the action can be re-written as

$$
\begin{aligned}
S_{\psi^{\prime}}= & \int \frac{d \omega^{\prime}}{2 \pi} \frac{d^{3} \mathbf{k}^{\prime}}{(2 \pi)^{3}} \psi_{a}^{\prime \dagger}\left(\omega^{\prime}, \mathbf{k}^{\prime}\right)\left\{i \omega^{\prime}-B^{\prime}\left[d_{1}\left(\mathbf{k}^{\prime}\right) \sigma_{1}\right.\right. \\
& \left.\left.+d_{2}\left(\mathbf{k}^{\prime}\right) \sigma_{2}\right]-A^{\prime} d_{3}\left(\mathbf{k}^{\prime}\right) \sigma_{3}\right\} \psi_{a}^{\prime}\left(\omega^{\prime}, \mathbf{k}^{\prime}\right),
\end{aligned}
$$

which recovers the original form of the action.

The free action of boson field $\phi$ takes the form

$$
S_{\phi}=\int \frac{d \omega}{2 \pi} \frac{d^{3} \mathbf{k}}{(2 \pi)^{3}} \phi(\omega, \mathbf{k})\left(k_{\perp}^{2}+\eta k_{z}^{2}\right) \phi(\omega, \mathbf{k}) .
$$

Including the interaction correction converts the action $\phi$ to

$$
\begin{aligned}
S_{\phi}= & \int \frac{d \omega}{2 \pi} \frac{d^{3} \mathbf{k}}{(2 \pi)^{3}} \phi(\omega, \mathbf{k})\left[k_{\perp}^{2}+\eta k_{z}^{2}+\Pi(0, \mathbf{k})\right] \phi(\omega, \mathbf{k}) \\
\approx & \int \frac{d \omega}{2 \pi} \frac{d^{3} \mathbf{k}}{(2 \pi)^{3}} \phi(\omega, \mathbf{k})\left[k_{\perp}^{2} e^{N C_{\perp} \ell}+\left(\eta+N C_{z} \ell\right) k_{z}^{2}\right] \\
& \times \phi(\omega, \mathbf{k}) .
\end{aligned}
$$

Making use of the transformations Eqs. (C3)- (C6), we find that the field $\phi$ should be re-scaled as follows

$$
\phi=\phi^{\prime} e^{-\frac{1}{2}\left(-\frac{17}{6}+N C_{\perp}\right) \ell} .
$$

Now the action of $\phi^{\prime}$ can be approximately written as

$$
\begin{aligned}
S_{\phi^{\prime}} \approx & \int \frac{d \omega^{\prime}}{2 \pi} \frac{d^{3} \mathbf{k}^{\prime}}{(2 \pi)^{3}} \phi^{\prime}\left(\omega^{\prime}, \mathbf{k}^{\prime}\right)\left\{k_{\perp}^{\prime 2}+\left[\eta-\eta\left(\frac{1}{3}+N C_{\perp}\right)\right.\right. \\
& \left.\left.\times \ell+N C_{z} \ell\right] k_{z}^{\prime 2}\right\} \phi^{\prime}\left(\omega^{\prime}, \mathbf{k}^{\prime}\right) .
\end{aligned}
$$

We define

$$
\eta^{\prime}=\eta-\eta\left(\frac{1}{3}+N C_{\perp}\right) \ell+N C_{z} \ell,
$$

and then get

$$
\begin{aligned}
S_{\phi^{\prime}}= & \int \frac{d \omega^{\prime}}{2 \pi} \frac{d^{3} \mathbf{k}^{\prime}}{(2 \pi)^{3}} \phi^{\prime}\left(\omega^{\prime}, \mathbf{k}^{\prime}\right)\left(k_{\perp}^{\prime 2}+\eta^{\prime} k_{z}^{\prime 2}\right) \\
& \times \phi^{\prime}\left(\omega^{\prime}, \mathbf{k}^{\prime}\right)
\end{aligned}
$$

which has the same form as the original boson action. 

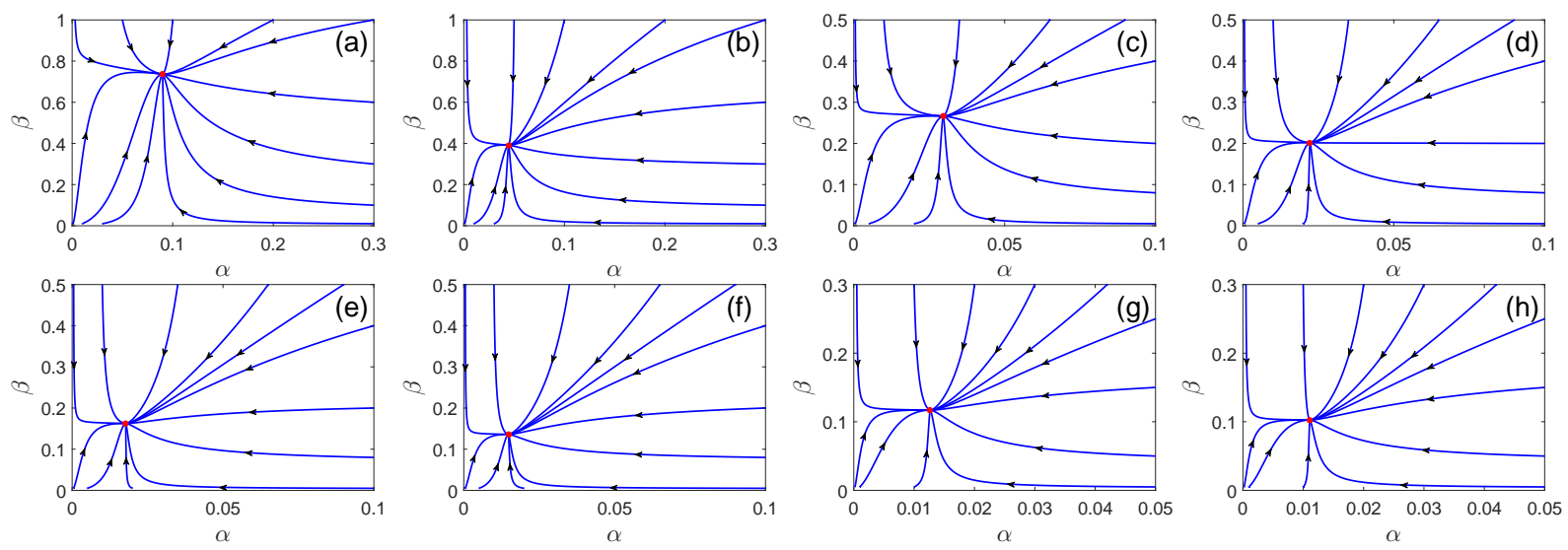

FIG. 5: (a)-(h): Flow diagrams on the $\alpha$ - $\beta$ plane at $N=1,2,3,4,5,6,7,8$, respectively.

The action of fermion-boson coupling is

$$
\begin{aligned}
S_{\psi \phi}= & g \int \frac{d \omega_{1}}{2 \pi} \frac{d^{3} \mathbf{k}_{1}}{(2 \pi)^{3}} \frac{d \omega_{2}}{2 \pi} \frac{d^{3} \mathbf{k}_{2}}{(2 \pi)^{3}} \psi_{a}^{\dagger}\left(\omega_{1}, \mathbf{k}_{1}\right) \psi_{a}\left(\omega_{2}, \mathbf{k}_{2}\right) \\
& \times \phi\left(\omega_{1}-\omega_{2}, \mathbf{k}_{1}-\mathbf{k}_{2}\right) .
\end{aligned}
$$

Making use of the transformations Eqs. (C3)-(C7) and (C13), we find that

$$
\begin{aligned}
S_{\psi^{\prime} \phi^{\prime}}= & g e^{\left(\frac{1}{4}-\frac{N C_{\perp}}{2}\right)} \ell \int \frac{d \omega_{1}^{\prime}}{2 \pi} \frac{d^{3} \mathbf{k}_{1}^{\prime}}{(2 \pi)^{3}} \frac{d \omega_{2}^{\prime}}{2 \pi} \frac{d^{3} \mathbf{k}_{2}^{\prime}}{(2 \pi)^{3}} \psi_{a}^{\prime \dagger}\left(\omega_{1}^{\prime}, \mathbf{k}_{1}^{\prime}\right) \\
& \times \psi_{a}^{\prime}\left(\omega_{2}^{\prime}, \mathbf{k}_{2}^{\prime}\right) \phi^{\prime}\left(\omega_{1}^{\prime}-\omega_{2}^{\prime}, \mathbf{k}_{1}^{\prime}-\mathbf{k}_{2}\right) . \quad(\mathrm{C} 18)
\end{aligned}
$$

Adopting the re-scaling transformation

$$
g^{\prime}=g e^{\left(\frac{1}{4}-\frac{N C_{\perp}}{2}\right) \ell},
$$

we further re-write the action in the form

$$
\begin{aligned}
S_{\psi^{\prime} \phi^{\prime}}= & g^{\prime} \int \frac{d \omega_{1}^{\prime}}{2 \pi} \frac{d^{3} \mathbf{k}_{1}^{\prime}}{(2 \pi)^{3}} \frac{d \omega_{2}^{\prime}}{2 \pi} \frac{d^{3} \mathbf{k}_{2}^{\prime}}{(2 \pi)^{3}} \psi_{a}^{\prime \dagger}\left(\omega_{1}^{\prime}, \mathbf{k}_{1}^{\prime}\right) \\
& \times \psi_{a}^{\prime}\left(\omega_{2}^{\prime}, \mathbf{k}_{2}^{\prime}\right) \phi_{a}^{\prime}\left(\omega_{1}^{\prime}-\omega_{2}^{\prime}, \mathbf{k}_{1}^{\prime}-\mathbf{k}_{2}^{\prime}\right),
\end{aligned}
$$

which recovers the form of the original interacting action.

From the transformations given by Eqs. (C8), (C9), (C15), and (C19), we obtain the following RG equations

$$
\begin{aligned}
\frac{d B}{d \ell} & =C_{2} B \\
\frac{d A}{d \ell} & =C_{3} A \\
\frac{d \alpha}{d \ell} & =\left(\frac{1}{2}-N C_{\perp}-\frac{C_{3}}{2}\right) \alpha \\
\frac{d \beta}{d \ell} & =\left(\frac{5}{6}+\frac{1}{2} C_{3}-\frac{2}{3} C_{2}-N \beta\right) \beta \\
\frac{d \eta}{d \ell} & =\left(-\frac{1}{3}-N C_{\perp}+N \beta\right) \eta \\
\frac{d \zeta}{d \ell} & =\left(-\frac{1}{3}+\frac{2}{3} C_{2}-C_{3}-N C_{\perp}+N \beta\right) \zeta
\end{aligned}
$$

where $\alpha$ and $\beta$ are defined as

$$
\begin{aligned}
& \alpha=\frac{g^{2}}{8 \pi^{3} \sqrt{A \Lambda}}, \\
& \beta=\frac{C_{z}}{\eta}=\frac{\Gamma\left(\frac{3}{4}\right) \Gamma\left(\frac{4}{3}\right) g^{2} \sqrt{A}}{24 \pi^{2} \Gamma\left(\frac{25}{12}\right) B^{2 / 3} \Lambda^{5 / 6} \eta} .
\end{aligned}
$$

As shown in Fig. 5. $(\alpha, \beta)$ always flows to a stable fixed point $\left(\alpha^{*}, \beta^{*}\right)$. The concrete values of $\alpha^{*}$ and $\beta^{*}$ are determined by the fermion flavor $N$. The values of $\alpha^{*}$, $\beta^{*}$, and several other parameters obtained for different fermion flavors are summarized in Table $\Pi$.

\section{Appendix D: Observable quantities in the free case}

Here, we will calculate a number of observable quantities, first in the non-interacting limit.

\section{Density of states}

The retarded fermion propagator takes the form

$$
G_{0}^{\mathrm{ret}}(\omega, \mathbf{k})=\frac{1}{\omega-\mathcal{H}_{f}(\mathbf{k})+i \delta}
$$

Its imaginary part can be easily obtained:

$$
\begin{aligned}
\operatorname{Im}\left[G_{0}^{\mathrm{ret}}(\omega, \mathbf{k})\right]= & -\pi \operatorname{sgn}(\omega)\left[\omega+\mathcal{H}_{f}(\mathbf{k})\right] \frac{1}{2 E_{\mathbf{k}}} \\
& \times\left[\delta\left(\omega+E_{\mathbf{k}}\right)+\delta\left(\omega-E_{\mathbf{k}}\right)\right] .
\end{aligned}
$$

The spectral function is given by

$$
\begin{aligned}
\mathcal{A}(\omega, \mathbf{k}) & =-\frac{1}{\pi} \operatorname{Tr}\left[\operatorname{Im}\left[G_{0}^{\mathrm{ret}}(\omega, \mathbf{k})\right]\right] \\
& =|\omega| \frac{1}{E_{\mathbf{k}}}\left[\delta\left(\omega+E_{\mathbf{k}}\right)+\delta\left(\omega-E_{\mathbf{k}}\right)\right],
\end{aligned}
$$


TABLE II: Dependence of the parameters $\alpha^{*}, \beta^{*}, C_{2}^{*}, C_{3}^{*}, C_{\perp}^{*}, N \alpha^{*}, N \beta^{*}, N C_{\perp}^{*}$ on $N$ at the stable fixed point.

\begin{tabular}{|c|c|c|c|c|c|c|c|c|}
\hline \hline$N$ & $\alpha^{*}$ & $\beta^{*}$ & $C_{2}^{*}$ & $C_{3}^{*}$ & $C_{\perp}^{*}$ & $N \alpha^{*}$ & $N \beta^{*}$ & $N C_{\perp}^{*}$ \\
\hline 1 & 0.08997 & 0.7364 & 0.1322 & -0.01754 & 0.5088 & 0.08997 & 0.7364 & 0.5088 \\
\hline 2 & 0.04462 & 0.3914 & 0.06891 & -0.009236 & 0.2523 & 0.08924 & 0.7828 & 0.5046 \\
\hline 3 & 0.02966 & 0.2664 & 0.04567 & -0.006258 & 0.1677 & 0.08897 & 0.7992 & 0.5031 \\
\hline 4 & 0.02221 & 0.2019 & 0.03517 & -0.004731 & 0.1256 & 0.08884 & 0.8075 & 0.5024 \\
\hline 5 & 0.01775 & 0.1625 & 0.02825 & -0.003802 & 0.1004 & 0.08876 & 0.8126 & 0.5019 \\
\hline 6 & 0.01478 & 0.1360 & 0.02361 & -0.003178 & 0.08360 & 0.08870 & 0.8160 & 0.5016 \\
\hline 7 & 0.01267 & 0.1169 & 0.02027 & -0.002730 & 0.07162 & 0.08866 & 0.8185 & 0.5014 \\
\hline 8 & 0.01108 & 0.1025 & 0.01776 & -0.002393 & 0.06265 & 0.08863 & 0.8203 & 0.5012 \\
\hline 9 & 0.009845 & 0.09130 & 0.01581 & -0.002130 & 0.05567 & 0.08861 & 0.8217 & 0.5010 \\
\hline 10 & 0.008859 & 0.08229 & 0.01424 & -0.001919 & 0.05010 & 0.08859 & 0.8229 & 0.5010 \\
\hline \hline
\end{tabular}

which directly leads to the fermion DOS

$$
\begin{aligned}
\rho(\omega)= & N \int \frac{d^{3} \mathbf{k}}{(2 \pi)^{3}} \mathcal{A}(\omega, \mathbf{k}) \\
= & \frac{|\omega|}{2 \pi^{2}} \int d k_{\perp} d\left|k_{z}\right| k_{\perp} \frac{1}{E_{\mathbf{k}}}\left[\delta\left(\omega+E_{\mathbf{k}}\right)\right. \\
& \left.+\delta\left(\omega-E_{\mathbf{k}}\right)\right] .
\end{aligned}
$$

After integrating over the momenta, we obtain

$$
\rho(\omega)=\frac{N c_{1}}{\sqrt{A} B^{2 / 3}}|\omega|^{1 / 6},
$$

where

$$
c_{1}=\frac{\Gamma\left(\frac{1}{3}\right) \Gamma\left(\frac{5}{4}\right)}{6 \pi^{2} \Gamma\left(\frac{7}{12}\right)} \approx 0.02682
$$

\section{Specific heat}

The Matsubara fermion propagator has the form

$$
\begin{aligned}
G_{0}\left(i \omega_{n}, \mathbf{k}\right) & =\frac{1}{i \omega_{n}-\mathcal{H}_{f}(\mathbf{k})} \\
& =\frac{-i \omega_{n}-\mathcal{H}_{f}(\mathbf{k})}{\omega_{n}^{2}+E_{\mathbf{k}}^{2}},
\end{aligned}
$$

where $\omega_{n}=(2 n+1) \pi T$ with $n$ being integers. The free energy of fermions is

$$
F_{f}(T)=-2 N T \sum_{\omega_{n}} \int \frac{d^{3} \mathbf{k}}{(2 \pi)^{3}} \ln \left[\left(\omega_{n}^{2}+E_{\mathbf{k}}^{2}\right)^{1 / 2}\right](\mathrm{D} 8)
$$

Performing frequency summation yields

$$
F_{f}(T)=-2 N \int \frac{d^{3} \mathbf{k}}{(2 \pi)^{3}}\left[E_{\mathbf{k}}+2 T \ln \left(1+e^{-\frac{E_{\mathbf{k}}}{T}}\right)\right]
$$

which is divergent due to the first term in bracket. To get a finite free energy, we replace $F_{f}(T)$ with $F_{f}(T)-F_{f}(0)$, and thus get

$$
\begin{aligned}
F_{f}(T) & =-4 N T \int \frac{d^{3} \mathbf{k}}{(2 \pi)^{3}} \ln \left(1+e^{-\frac{E_{\mathbf{k}}}{T}}\right) \\
& =-\frac{2 N T}{\pi^{2}} \int d k_{\perp} d\left|k_{z}\right| k_{\perp} \ln \left(1+e^{-\frac{E_{\mathbf{k}}}{T}}\right)(\mathrm{D} 10)
\end{aligned}
$$

Employing the transformations Eqs. A99-A11 and then carrying out the integrations, we have

$$
\begin{aligned}
F_{f}(T)= & -\frac{\left(4-2^{5 / 6}\right) \zeta\left(\frac{13}{6}\right) \Gamma\left(\frac{7}{6}\right) \Gamma\left(\frac{1}{3}\right) \Gamma\left(\frac{5}{4}\right) N}{6 \pi^{2} \Gamma\left(\frac{7}{12}\right) \sqrt{A} B^{2 / 3}} \\
& \times T^{13 / 6}
\end{aligned}
$$

where $\zeta(x)$ is the Riemann zeta function. The corresponding specific heat satisfies

$$
C_{v}(T)=-T \frac{\partial^{2} F_{f}(T)}{\partial T^{2}}=\frac{N c_{2}}{\sqrt{A} B^{2 / 3}} T^{7 / 6},
$$

where

$$
\begin{aligned}
c_{2} & =\frac{91\left(4-2^{5 / 6}\right) \zeta\left(\frac{13}{6}\right) \Gamma\left(\frac{7}{6}\right) \Gamma\left(\frac{1}{3}\right) \Gamma\left(\frac{5}{4}\right)}{216 \pi^{2} \Gamma\left(\frac{7}{12}\right)} \\
& \approx 0.211 .
\end{aligned}
$$

\section{Compressibility}

In order to calculate the compressibility, we formally include a finite chemical potential $\mu$ into the fermion propagator, namely

$$
G_{0}\left(i \omega_{n}, \mathbf{k}\right)=\frac{-i \omega_{n}-\mu-\mathcal{H}_{f}(\mathbf{k})}{\left(\omega_{n}-i \mu\right)^{2}+E_{\mathbf{k}}^{2}} .
$$

The corresponding free energy now becomes

$$
\begin{aligned}
F_{f}(T, \mu)= & -2 N T \sum_{\omega_{n}} \int \frac{d^{3} \mathbf{k}}{(2 \pi)^{3}} \\
& \times \ln \left[\left(\left(\omega_{n}-i \mu\right)^{2}+E_{\mathbf{k}}^{2}\right)^{1 / 2}\right] .
\end{aligned}
$$


Summing over frequencies allows us to get

$$
\begin{aligned}
F_{f}(T, \mu)= & -2 N T \int \frac{d^{3} \mathbf{k}}{(2 \pi)^{3}}\left[\ln \left(1+e^{-\frac{E_{\mathbf{k}}-\mu}{T}}\right)\right. \\
& \left.+\ln \left(1+e^{-\frac{E_{\mathbf{k}}+\mu}{T}}\right)\right] \\
= & -\frac{N T}{\pi^{2}} \int d k_{\perp} d\left|k_{z}\right| k_{\perp}\left[\ln \left(1+e^{-\frac{E_{\mathbf{k}}-\mu}{T}}\right)\right. \\
& \left.+\ln \left(1+e^{-\frac{E_{\mathbf{k}}+\mu}{T}}\right)\right] .
\end{aligned}
$$

Using the transformations Eqs. (A9)-A11 and carrying out integration over momenta, we obtain

$$
\begin{aligned}
F_{f}(T, \mu)= & \frac{N \Gamma\left(\frac{1}{3}\right)\left(\frac{5}{4}\right) \Gamma\left(\frac{7}{6}\right)}{3 \pi^{2} \Gamma\left(\frac{7}{12}\right) \sqrt{A} B^{2 / 3}} T^{13 / 6}\left[\operatorname{Li}_{\frac{13}{6}}\left(-e^{\frac{\mu}{T}}\right)\right. \\
& \left.+\operatorname{Li}_{\frac{13}{6}}\left(-e^{-\frac{\mu}{T}}\right)\right]
\end{aligned}
$$

where $\operatorname{Li}_{x}(y)$ is the polylogarithmic function. The compressibility is

$$
\begin{aligned}
\kappa(T, \mu)= & -\frac{\partial^{2} F_{f}(T, \mu)}{\partial \mu^{2}} \\
= & -\frac{N \Gamma\left(\frac{1}{3}\right)\left(\frac{5}{4}\right) \Gamma\left(\frac{7}{6}\right)}{3 \pi^{2} \Gamma\left(\frac{7}{12}\right) \sqrt{A} B^{2 / 3}} T^{1 / 6}\left[\operatorname{Li}_{\frac{1}{6}}\left(-e^{\frac{\mu}{T}}\right)\right. \\
& \left.+\operatorname{Li}_{\frac{1}{6}}\left(-e^{-\frac{\mu}{T}}\right)\right] .
\end{aligned}
$$

Taking the limit $\mu=0$, we finally get

$$
\kappa(T)=\frac{N c_{3}}{\sqrt{A} B^{2 / 3}} T^{1 / 6},
$$

where

$$
\begin{aligned}
c_{3} & =\frac{2\left(1-2^{5 / 6}\right) \zeta\left(\frac{1}{6}\right) \Gamma\left(\frac{1}{3}\right) \Gamma\left(\frac{5}{4}\right) \Gamma\left(\frac{7}{6}\right)}{3 \pi^{2} \Gamma\left(\frac{7}{12}\right)} \\
& \approx 0.05343 .
\end{aligned}
$$

\section{Dynamical conductivities}

The dynamical conductivities can be obtained by calculating the current-current correlation function, which in the Matsubara formalism is usually defined as

$$
\begin{aligned}
\Pi_{i j}\left(i \Omega_{m}\right)= & -e^{2} T \sum_{\omega_{n}} \int \frac{d^{3} \mathbf{k}}{(2 \pi)^{3}} \operatorname{Tr}\left[\gamma_{i}(\mathbf{k}) G_{0}\left(i \omega_{n}, \mathbf{k}\right) \gamma_{j}(\mathbf{k})\right. \\
& \left.\times G_{0}\left(i \omega_{n}+i \Omega_{m}, \mathbf{k}\right)\right], \quad(\mathrm{D} 21)
\end{aligned}
$$

where $\Omega_{m}=2 m \pi T$ with $m$ being integers. Here, $\gamma_{i}$ is given by

It is easy to verify that

$$
\gamma_{i}=\frac{\partial \mathcal{H}_{f}}{\partial k_{i}}
$$

$$
\begin{aligned}
& \gamma_{x}=\frac{\partial \mathcal{H}_{f}}{\partial k_{x}}=3 B\left(k_{x}^{2}-k_{y}^{2}\right) \sigma_{1}-6 B k_{x} k_{y} \sigma_{2}, \quad(\mathrm{D} 23) \\
& \gamma_{y}=\frac{\partial \mathcal{H}_{f}}{\partial k_{y}}=-6 B k_{x} k_{y} \sigma_{1}+3 B\left(k_{y}^{2}-k_{x}^{2}\right) \sigma_{2},(\mathrm{D} 24) \\
& \gamma_{z}=\frac{\partial \mathcal{H}_{f}}{\partial k_{z}}=2 A k_{z} \sigma_{3} .
\end{aligned}
$$

The symmetry of the Hamiltonian dictates that

$$
\Pi_{x x}=\Pi_{y y} \equiv \Pi_{\perp \perp},
$$

so it is only necessary to calculate $\Pi_{x x}$ and $\Pi_{z z}$, defined as follows

$$
\begin{aligned}
\Pi_{x x}\left(i \Omega_{m}\right)= & -e^{2} T \sum_{\omega_{n}} \int \frac{d^{3} \mathbf{k}}{(2 \pi)^{3}} \operatorname{Tr}\left[\gamma_{x} G_{0}\left(i \omega_{n}, \mathbf{k}\right) \gamma_{x}\right. \\
& \left.\times G_{0}\left(i \omega_{n}+i \Omega_{m}, \mathbf{k}\right)\right], \\
\Pi_{z z}\left(i \Omega_{m}\right)= & -e^{2} T \sum_{\omega_{n}} \int \frac{d^{3} \mathbf{k}}{(2 \pi)^{3}} \operatorname{Tr}\left[\gamma_{z} G_{0}\left(i \omega_{n}, \mathbf{k}\right) \gamma_{z}\right. \\
& \left.\times G_{0}\left(i \omega_{n}+i \Omega_{m}, \mathbf{k}\right)\right] .
\end{aligned}
$$

With the help of spectral representation

$$
G_{0}\left(i \omega_{n}, \mathbf{k}\right)=-\int_{-\infty}^{+\infty} \frac{d \omega_{1}}{\pi} \frac{\operatorname{Im}\left[G_{0}^{\mathrm{ret}}\left(\omega_{1}, \mathbf{k}\right)\right]}{i \omega_{n}-\omega_{1}}
$$

we now can re-write $\Pi_{x x}$ and $\Pi_{z z}$ as follows

$$
\begin{aligned}
\Pi_{x x}\left(i \Omega_{m}\right)= & -9 B^{2} e^{2} \int \frac{d^{3} \mathbf{k}}{(2 \pi)^{3}} \int_{-\infty}^{+\infty} \frac{d \omega_{1}}{\pi} \int_{-\infty}^{+\infty} \frac{d \omega_{2}}{\pi}\left\{\left(k_{x}^{2}-k_{y}^{2}\right)^{2} \operatorname{Tr}\left[\sigma_{1} \operatorname{Im}\left[G_{0}^{\mathrm{ret}}\left(\omega_{1}, \mathbf{k}\right)\right] \sigma_{1} \operatorname{Im}\left[G_{0}^{\mathrm{ret}}\left(\omega_{2}, \mathbf{k}\right)\right]\right]\right. \\
& \left.+4 k_{x}^{2} k_{y}^{2} \operatorname{Tr}\left[\sigma_{2} \operatorname{Im}\left[G_{0}^{\mathrm{ret}}\left(\omega_{1}, \mathbf{k}\right)\right] \sigma_{2} \operatorname{Im}\left[G_{0}^{\mathrm{ret}}\left(\omega_{2}, \mathbf{k}\right)\right]\right]\right\} \frac{n_{F}\left(\omega_{1}\right)-n_{F}\left(\omega_{2}\right)}{\omega_{1}-\omega_{2}+i \Omega_{m}} \\
\Pi_{z z}\left(i \Omega_{m}\right)= & -4 A^{2} e^{2} \int \frac{d^{3} \mathbf{k}}{(2 \pi)^{3}} k_{z}^{2} \int_{-\infty}^{+\infty} \frac{d \omega_{1}}{\pi} \int_{-\infty}^{+\infty} \frac{d \omega_{2}}{\pi} \operatorname{Tr}\left[\sigma_{3} \operatorname{Im}\left[G_{0}^{\mathrm{ret}}\left(\omega_{1}, \mathbf{k}\right)\right] \sigma_{3} \operatorname{Im}\left[G_{0}^{\mathrm{ret}}\left(\omega_{2}, \mathbf{k}\right)\right]\right] \\
& \times \frac{n_{F}\left(\omega_{1}\right)-n_{F}\left(\omega_{2}\right)}{\omega_{1}-\omega_{2}+i \Omega_{m}}
\end{aligned}
$$


where $n_{F}(x)=\frac{1}{e^{x / T}+1}$. We then carry out analytical continuation $i \Omega_{m} \rightarrow \Omega+i \delta$, and get the imaginary parts:

$$
\begin{aligned}
\operatorname{Im}\left[\Pi_{x x}^{\mathrm{ret}}(\Omega, T)\right]= & 9 B^{2} e^{2} \int \frac{d^{3} \mathbf{k}}{(2 \pi)^{3}} \int_{-\infty}^{+\infty} \frac{d \omega_{1}}{\pi}\left\{\left(k_{x}^{2}-k_{y}^{2}\right)^{2} \operatorname{Tr}\left[\sigma_{1} \operatorname{Im}\left[G_{0}^{\mathrm{ret}}\left(\omega_{1}, \mathbf{k}\right)\right] \sigma_{1} \operatorname{Im}\left[G_{0}^{\mathrm{ret}}\left(\omega_{1}+\Omega, \mathbf{k}\right)\right]\right]\right. \\
& \left.+4 k_{x}^{2} k_{y}^{2} \operatorname{Tr}\left[\sigma_{2} \operatorname{Im}\left[G_{0}^{\mathrm{ret}}\left(\omega_{1}, \mathbf{k}\right)\right] \sigma_{2} \operatorname{Im}\left[G_{0}^{\mathrm{ret}}\left(\omega_{1}+\Omega, \mathbf{k}\right)\right]\right]\right\}\left[n_{F}\left(\omega_{1}\right)-n_{F}\left(\omega_{1}+\Omega\right)\right], \\
\operatorname{Im}\left[\Pi_{z z}^{\mathrm{ret}}(\Omega, T)\right]= & 4 A^{2} e^{2} \int \frac{d^{3} \mathbf{k}}{(2 \pi)^{3}} k_{z}^{2} \int_{-\infty}^{+\infty} \frac{d \omega_{1}}{\pi} \operatorname{Tr}\left[\sigma_{3} \operatorname{Im}\left[G_{0}^{\mathrm{ret}}\left(\omega_{1}, \mathbf{k}\right)\right] \sigma_{3} \operatorname{Im}\left[G_{0}^{\mathrm{ret}}\left(\omega_{1}+\Omega, \mathbf{k}\right)\right]\right] \\
& \times\left[n_{F}\left(\omega_{1}\right)-n_{F}\left(\omega_{1}+\Omega\right)\right] .
\end{aligned}
$$

The formula $\frac{1}{x+i \delta}=\mathcal{P} \frac{1}{x}-i \pi \delta(x)$, where $\mathcal{P} \frac{1}{x}$ is the principal value, was used above. The conductivities are related to current-current correlation function as follows

$$
\begin{aligned}
\sigma_{x x}(\Omega, T) & =\frac{\operatorname{Im}\left[\Pi_{x x}^{\mathrm{ret}}(\Omega, T)\right]}{\Omega}, \\
\sigma_{z z}(\Omega, T) & =\frac{\operatorname{Im}\left[\Pi_{z z}^{\mathrm{ret}}(\Omega, T)\right]}{\Omega} .
\end{aligned}
$$

After accomplishing tedious but straightforward calculations, we eventually obtain

$$
\begin{aligned}
\sigma_{x x}(\Omega, T)= & \frac{3 e^{2}}{4 \pi \sqrt{A}} \delta(\Omega) T^{3 / 2} \int_{0}^{+\infty} d x x^{3 / 2} \frac{1}{\sinh ^{2}\left(\frac{x}{2}\right)} \\
& +\frac{c_{4} e^{2}}{\sqrt{A}}|\Omega|^{1 / 2} \tanh \left(\frac{|\Omega|}{4 T}\right), \quad \text { (D35) } \\
\sigma_{z z}(\Omega, T)= & \frac{36 \Gamma\left(\frac{1}{3}\right) \Gamma\left(\frac{3}{4}\right) \sqrt{A} e^{2}}{13 \Gamma\left(\frac{1}{12}\right) \pi B^{2 / 3}} \delta(\Omega) T^{7 / 6} \\
& \times \int_{0}^{+\infty} d x x^{7 / 6} \frac{1}{\sinh ^{2}\left(\frac{x}{2}\right)} \\
& +\frac{c_{5} \sqrt{A} e^{2}}{B^{2 / 3}}|\Omega|^{1 / 6} \tanh ^{\left(\frac{|\Omega|}{4 T}\right),}
\end{aligned}
$$

where

$$
\begin{aligned}
& c_{4}=\frac{9}{8 \sqrt{2} \pi} \approx 0.2532, \\
& c_{5}=\frac{2^{5 / 6} \Gamma\left(\frac{1}{3}\right) \Gamma\left(\frac{3}{4}\right)}{13 \Gamma\left(\frac{1}{12}\right) \pi} \approx 0.01245 .
\end{aligned}
$$

The first terms in the right-hand side of Eqs. (D35) and (D36) represent the Drude peak.

\section{Diamagnetic Susceptibilities}

The diamagnetic susceptibility is defined as

$$
\begin{aligned}
\chi_{D}= & -N e^{2} T \sum_{\omega_{n}} \int \frac{d^{3} \mathbf{k}}{(2 \pi)^{3}} \operatorname{Tr}\left[\gamma_{a} G_{0}\left(i \omega_{n}, \mathbf{k}\right) \gamma_{b}\right. \\
& \left.\times G_{0}\left(i \omega_{n}, \mathbf{k}\right) \gamma_{a} G_{0}\left(i \omega_{n}, \mathbf{k}\right) \gamma_{b} G_{0}\left(i \omega_{n}, \mathbf{k}\right)\right](\mathrm{D} 38)
\end{aligned}
$$

Here, $\gamma_{a}$ is given by

$$
\gamma_{a}=\frac{\partial \mathcal{H}_{f}}{\partial k_{a}}
$$

where $a$ denotes an axis that is perpendicular to the direction of external magnetic field. Owing to the rotational symmetry of the system, the diamagnetic susceptibility should exhibit the same behavior for fields aligned along $x$ and $y$ axes, i.e., $\chi_{D}^{x}=\chi_{D}^{y} \equiv \chi_{D}^{\perp}$. However, for magnetic field $\mathbf{B}=B \mathbf{e}_{z}$, diamagnetic susceptibility should be different. By taking $\mathbf{B}=B \mathbf{e}_{x}$ as an example, we express the diamagnetic susceptibility in the form

$$
\begin{aligned}
\chi_{D}^{\perp}= & -N e^{2} T \sum_{\omega_{n}} \int \frac{d^{3} \mathbf{k}}{(2 \pi)^{3}} \operatorname{Tr}\left[\gamma_{y} G_{0}\left(i \omega_{n}, \mathbf{k}\right) \gamma_{z} G_{0}\left(i \omega_{n}, \mathbf{k}\right)\right. \\
& \left.\times \gamma_{y} G_{0}\left(i \omega_{n}, \mathbf{k}\right) \gamma_{z} G_{0}\left(i \omega_{n}, \mathbf{k}\right)\right] .
\end{aligned}
$$

In the case of magnetic field $\mathbf{B}=B \mathbf{e}_{z}$, the diamagnetic susceptibility is defined as

$$
\begin{aligned}
\chi_{D}^{z}= & -N e^{2} T \sum_{\omega_{n}} \int \frac{d^{3} \mathbf{k}}{(2 \pi)^{3}} \operatorname{Tr}\left[\gamma_{x} G_{0}\left(i \omega_{n}, \mathbf{k}\right) \gamma_{y} G_{0}\left(i \omega_{n}, \mathbf{k}\right)\right. \\
& \left.\times \gamma_{x} G_{0}\left(i \omega_{n}, \mathbf{k}\right) \gamma_{y} G_{0}\left(i \omega_{n}, \mathbf{k}\right)\right], \quad(\mathrm{D} 41)
\end{aligned}
$$

where $\gamma_{x}, \gamma_{y}$, and $\gamma_{z}$ are given by the Eqs. (D23)(D25). Substituting the fermion propagator and $\gamma_{i}$ into Eqs. (D40) and (D41), and performing integration over the azimuthal angle within the $x-y$ plane, we obtain

$$
\begin{aligned}
\chi_{D}^{\perp}= & N A^{2} B^{2} e^{2} T \frac{36}{\pi^{2}} \int d k_{\perp} d\left|k_{z}\right| k_{\perp}^{5} k_{z}^{2} \\
& \times\left(S_{A}-4 B^{2} k_{\perp}^{6} A^{2} k_{z}^{4} S_{B}\right), \\
\chi_{D}^{z}= & N B^{4} e^{2} T \frac{81}{\pi^{2}} \int d k_{\perp} d\left|k_{z}\right| k_{\perp}^{9} \\
& \times\left(S_{A}-B^{4} k_{\perp}^{12} S_{B}\right) .
\end{aligned}
$$

where

$$
\begin{aligned}
& S_{A}=\sum_{\omega_{n}} \frac{1}{\left(\omega_{n}^{2}+E_{\mathbf{k}}^{2}\right)^{2}}, \\
& S_{B}=\sum_{\omega_{n}} \frac{1}{\left(\omega_{n}^{2}+E_{\mathbf{k}}^{2}\right)^{4}} .
\end{aligned}
$$

After carrying out frequency summation, $S_{A}$ and $S_{B}$ can be further written as

$$
\left.S_{A}=\frac{1}{(2 \pi T)^{4}} \frac{1}{2 Y}\left[\frac{\pi}{Y^{2}} \tanh (\pi Y)-\frac{\pi^{2}}{Y} \frac{1}{\cosh ^{2}(\pi Y)}\right] \mathrm{D} 46\right)
$$




$$
\begin{aligned}
S_{B}= & \frac{1}{(2 \pi T)^{8}} \frac{1}{48 Y^{3}}\left[\frac{15 \pi}{Y^{4}} \tanh (\pi Y)-\frac{15 \pi^{2}}{Y^{3}} \frac{1}{\cosh ^{2}(\pi Y)}\right. \\
& -\frac{12 \pi^{3}}{Y^{2}} \frac{\tanh (\pi Y)}{\cosh ^{2}(\pi Y)}+\frac{2 \pi^{4}}{Y} \frac{1}{\cosh ^{4}(\pi Y)} \\
& \left.-\frac{4 \pi^{4}}{Y} \frac{\tanh ^{2}(\pi Y)}{\cosh ^{2}(\pi Y)}\right],
\end{aligned}
$$

where $Y=\frac{E_{\mathrm{k}}}{2 \pi T}$. Substituting Eqs. (D46) and (D47) into Eqs. (D42) and (D43), and then invoking transformations Eqs. A9 - A11 , followed by an integration over $\xi$, we find that the following diamagnetic susceptibilities

$$
\begin{aligned}
& \chi_{D}^{\perp}=N c_{\chi^{\perp}} \sqrt{A} e^{2} \sqrt{T}, \\
& \chi_{D}^{z}=N c_{\chi_{z}} \frac{B^{2 / 3}}{\sqrt{A}} e^{2} T^{5 / 6},
\end{aligned}
$$

where

$$
\begin{aligned}
c_{\chi^{\perp}}= & \frac{2}{77 \sqrt{2} \pi^{2}} \int_{0}^{+\infty} d x\left[\frac{47}{x^{1 / 2}} \tanh (x)-47 \frac{x^{1 / 2}}{\cosh ^{2}(x)}\right. \\
& +24 x^{3 / 2} \frac{\tanh (x)}{\cosh ^{2}(x)}-4 x^{5 / 2} \frac{1}{\cosh ^{4}(x)} \\
& \left.+8 x^{5 / 2} \frac{\tanh ^{2}(x)}{\cosh ^{2}(x)}\right], \\
c_{\chi_{z}}= & \frac{\Gamma\left(\frac{5}{4}\right) \Gamma\left(\frac{5}{3}\right)}{322 \pi^{2} 2^{1 / 6} \Gamma\left(\frac{23}{12}\right)} \int_{0}^{+\infty} d x x^{11 / 6}\left[\frac{2187}{x^{2}} \tanh (x)\right.
\end{aligned}
$$

$$
\begin{aligned}
c_{6}= & -\frac{188}{77 \sqrt{2} \pi^{2}}+\frac{94}{77 \sqrt{2} \pi^{2}} \int_{1}^{+\infty} d x\left[\frac{1}{x^{1 / 2}} \tanh (x)-\frac{1}{x^{1 / 2}}\right]+\frac{94}{77 \sqrt{2} \pi^{2}} \int_{0}^{1} d x \frac{1}{x^{1 / 2}} \tanh (x) \\
& +\frac{2}{77 \sqrt{2} \pi^{2}} \int_{0}^{+\infty} d x\left[-47 x^{1 / 2} \frac{1}{\cosh ^{2}(x)}+24 x^{3 / 2} \frac{\tanh (x)}{\cosh ^{2}(x)}-4 x^{5 / 2} \frac{1}{\cosh ^{4}(x)}+8 x^{5 / 2} \frac{\tanh ^{2}(x)}{\cosh ^{2}(x)}\right]
\end{aligned}
$$$$
\approx 0.1869 \text {, }
$$$$
c_{7}=-\frac{13122 \Gamma\left(\frac{5}{4}\right) \Gamma\left(\frac{5}{3}\right)}{1610 \pi^{2} 2^{1 / 6} \Gamma\left(\frac{23}{12}\right)}+\frac{2187 \Gamma\left(\frac{5}{4}\right) \Gamma\left(\frac{5}{3}\right)}{322 \pi^{2} 2^{1 / 6} \Gamma\left(\frac{23}{12}\right)} \int_{1}^{+\infty} d x\left[\frac{1}{x^{1 / 6}} \tanh (x)-\frac{1}{x^{1 / 6}}\right]+\frac{2187 \Gamma\left(\frac{5}{4}\right) \Gamma\left(\frac{5}{3}\right)}{322 \pi^{2} 2^{1 / 6} \Gamma\left(\frac{23}{12}\right)} \int_{0}^{1} d x \frac{1}{x^{1 / 6}} \tanh (x)
$$$$
+\frac{\Gamma\left(\frac{5}{4}\right) \Gamma\left(\frac{5}{3}\right)}{322 \pi^{2} 2^{1 / 6} \Gamma\left(\frac{23}{12}\right)} \int_{0}^{+\infty} d x x^{11 / 6}\left[-\frac{2187}{x} \frac{1}{\cosh ^{2}(x)}+1728 \frac{\tanh (x)}{\cosh ^{2}(x)}-288 x \frac{1}{\cosh ^{4}(x)}+576 x \frac{\tanh ^{2}(x)}{\cosh ^{2}(x)}\right]
$$$$
\approx-0.4131 \text {. }
$$

$$
\begin{aligned}
\chi_{D 0}^{\perp} & =N \frac{94}{77 \pi^{2}} \sqrt{A} e^{2} \sqrt{\Lambda}, \\
\chi_{D 0}^{z} & =N \frac{6561 \Gamma\left(\frac{5}{4}\right) \Gamma\left(\frac{5}{3}\right)}{1610 \pi^{2} \Gamma\left(\frac{23}{12}\right)} \frac{B^{2 / 3}}{\sqrt{A}} e^{2} \Lambda^{5 / 6},
\end{aligned}
$$

and

where

$$
\begin{aligned}
& -\frac{1}{x} \frac{2187}{\cosh ^{2}(x)}+1728 \frac{\tanh (x)}{\cosh ^{2}(x)}-\frac{288 x}{\cosh ^{4}(x)} \\
& \left.+576 x \frac{\tanh ^{2}(x)}{\cosh ^{2}(x)}\right] .
\end{aligned}
$$

Here, a transformation $E=2 T x$ has been used. Notice of the integrands of Eqs. (D50) and (D51) are actually divergent. To regularize the integral, we need to introduce a cutoff $\frac{\Lambda}{2 T}$. After doing so, we find that the low- $T$

$$
\begin{aligned}
& \chi_{\frac{\perp}{D}}^{\perp}=\chi_{D 0}^{\perp}+N c_{6} \sqrt{A} e^{2} \sqrt{T}, \\
& \chi_{D}^{z}=\chi_{D 0}^{z}+N c_{7} \frac{B^{2 / 3}}{\sqrt{A}} e^{2} T^{5 / 6},
\end{aligned}
$$

\section{(1)}


corrections, we get

$$
\frac{d \ln (\rho)}{d \ln (\omega)} \sim \frac{1}{6}+\frac{\ln \left(\frac{1}{\sqrt{A} B^{2 / 3}}\right)}{d \ln (\omega)} .
$$

We have got the RG equations of $A$ and $B$ :

$$
\begin{aligned}
& \frac{d A}{d \ell}=C_{3} A, \\
& \frac{d B}{d \ell}=C_{2} B .
\end{aligned}
$$

Using the transformation $\omega=\omega_{0} e^{-\ell}$, we find that $A$ and $B$ depend on energy $\omega$ as follows

$$
\begin{aligned}
& \frac{d \ln (A)}{d \ln (\omega)}=-C_{3}, \\
& \frac{d \ln (B)}{d \ln (\omega)}=-C_{2},
\end{aligned}
$$

Substituting Eqs. (E5) and (E6) into Eq. (E2), we have

$$
\frac{d \ln (\rho)}{d \ln (\omega)} \sim \frac{1}{6}+\frac{1}{2} C_{3}+\frac{2}{3} C_{2} .
$$

It can be further approximately written as

$$
\frac{d \ln (\rho)}{d \ln (\omega)} \sim \frac{1}{6}+\frac{1}{2} C_{3}^{*}+\frac{2}{3} C_{2}^{*},
$$

which has the following solution

$$
\rho(\omega) \sim \omega^{\frac{1}{6}+\frac{1}{2} C_{3}^{*}+\frac{2}{3} C_{2}^{*}} \sim \omega^{1 / 6+\eta_{1}}
$$

where

$$
\eta_{1}=\frac{2}{3} C_{2}^{*}+\frac{1}{2} C_{3}^{*}
$$

\section{Specific heat}

In the non-interacting limit, we have found the specific heat

$$
C_{v} \sim \frac{T^{7 / 6}}{\sqrt{A} B^{2 / 3}}
$$

Once the interaction corrections are taken into account, parameters $A$ and $B$ become $T$-dependent at finite $T$. One can verify that

$$
\frac{d C_{v}}{d T}=\frac{7}{6}+\frac{\ln \left(\frac{1}{\sqrt{A} B^{2 / 3}}\right)}{d \ln (T)} .
$$

Using the transformation $T=T_{0} e^{-\ell}$ where $T_{0}$ is an initial value, we use Eqs. (E3) and (E4) to obtain

$$
\begin{aligned}
& \frac{d \ln (A)}{d \ln (T)}=-C_{3}, \\
& \frac{d \ln (B)}{d \ln (T)}=-C_{2} .
\end{aligned}
$$

Substituting Eqs. (E13) and (E14) into Eq. (E12), we get

$$
\begin{aligned}
\frac{d \ln \left(C_{v}\right)}{d \ln (T)} & \sim \frac{7}{6}+\frac{2}{3} C_{2}+\frac{1}{2} C_{3} \\
& \sim \frac{7}{6}+\frac{2}{3} C_{2}^{*}+\frac{1}{2} C_{3}^{*},
\end{aligned}
$$

which leads to the renormalized specific heat

$$
C_{v}(T) \sim T^{\frac{7}{6}+\frac{2}{3} C_{2}^{*}+\frac{1}{2} C_{3}^{*}} \sim T^{7 / 6+\eta_{1}} .
$$

\section{Compressibility}

In the non-interacting limit, the compressibility is

$$
\kappa \sim \frac{1}{\sqrt{A} B^{2 / 3}} T^{1 / 6} .
$$

After including interaction corrections, we obtain

$$
\frac{d \ln (\kappa)}{d \ln (T)} \sim \frac{1}{6}+\frac{d \ln \left(\frac{1}{\sqrt{A} B^{2 / 3}}\right)}{d \ln (T)} .
$$

Substituting Eqs. (E13) and (E14) into Eq. (E17) leads to

$$
\begin{aligned}
\frac{d \ln (\kappa)}{d \ln (T)} & \sim \frac{1}{6}+\frac{2}{3} C_{2}+\frac{1}{2} C_{3} \\
& \sim \frac{1}{6}+\frac{2}{3} C_{2}^{*}+\frac{1}{2} C_{3}^{*} .
\end{aligned}
$$

Now the renormalized compressibility can be written as

$$
\kappa(T) \sim T^{\frac{1}{6}+\frac{2}{3} C_{2}^{*}+\frac{1}{2} C_{3}^{*}} \sim T^{1 / 6+\eta_{1}} .
$$

\section{Conductivities}

In the free case, the conductivities within the $x-y$ plane and along $z$-axis are given by

$$
\sigma_{\perp \perp} \sim \frac{e^{2}}{\sqrt{A}} \omega^{1 / 2}, \quad \text { and } \quad \sigma_{z z} \sim \frac{\sqrt{A} e^{2}}{B^{2 / 3}} \omega^{1 / 6} .
$$

Incorporating the interaction corrections of $A, B$, and $e$, we similarly have

$$
\begin{aligned}
& \frac{d \ln \left(\sigma_{\perp \perp}\right)}{d \ln (\omega)} \sim \frac{1}{2}+\frac{d \ln \left(\frac{e^{2}}{\sqrt{A}}\right)}{d \ln (\omega)}, \\
& \frac{d \ln \left(\sigma_{z z}\right)}{d \ln (\omega)} \sim \frac{1}{6}+\frac{d \ln \left(\frac{\sqrt{A} e^{2}}{B^{2 / 3}}\right)}{d \ln (\omega)} .
\end{aligned}
$$

From the definition $g=\frac{\sqrt{4 \pi} e}{\epsilon}$ and the RG equation

$$
\frac{d g}{d \ell}=\left(\frac{1}{4}-\frac{N C_{\perp}}{2}\right) g
$$


we get

$$
\frac{d e}{d \ell}=\left(\frac{1}{4}-\frac{N C_{\perp}}{2}\right) e .
$$

Once again we utilize the transformation $\omega=\omega_{0} e^{-\ell}$ to obtain

$$
\frac{d \ln (e)}{d \ln (\omega)}=-\left(\frac{1}{4}-\frac{N C_{\perp}}{2}\right) .
$$

Substituting Eqs. (E5), (E6), and (E25) into Eqs. (E21) and (E22), we find that the conductivities satisfy the equations as follows

$$
\begin{aligned}
\frac{d \ln \left(\sigma_{\perp \perp}\right)}{d \ln (\omega)} & \sim \frac{1}{2}-\frac{1}{2}+N C_{\perp}+\frac{1}{2} C_{3} \\
& \sim \frac{1}{2}-\frac{1}{2}+N C_{\perp}^{*}+\frac{1}{2} C_{3}^{*}, \\
\frac{d \ln \left(\sigma_{z z}\right)}{d \ln (\omega)} & \sim \frac{1}{6}-\frac{1}{2}+N C_{\perp}+\frac{2}{3} C_{2}-\frac{1}{2} C_{3} \\
& \sim \frac{1}{6}-\frac{1}{2}+N C_{\perp}^{*}+\frac{2}{3} C_{2}^{*}-\frac{1}{2} C_{3}^{*} .
\end{aligned}
$$

Solving these two equations, we now obtain the renormalized conductivities

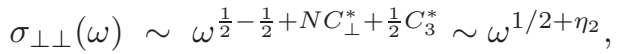

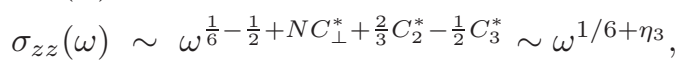

where

$$
\begin{aligned}
& \eta_{2}=-\frac{1}{2}+N C_{\perp}^{*}+\frac{1}{2} C_{3}^{*}=0 \\
& \eta_{3}=-\frac{1}{2}+N C_{\perp}^{*}+\frac{2}{3} C_{2}^{*}-\frac{1}{2} C_{3}^{*} .
\end{aligned}
$$

We see that $\sigma_{\perp \perp}(\omega)$ remains qualitatively intact after including the interaction corrections, whereas $\sigma_{z z}(\omega)$ is considerably altered.

TABLE III: Dependence of $\eta_{1}, \eta_{2}, \eta_{3}, \eta_{4}$, and $\eta_{5}$ on fermion flavor $N$.

\begin{tabular}{|c|c|c|c|c|c|}
\hline \hline$N$ & $\eta_{1}$ & $\eta_{2}$ & $\eta_{3}$ & $\eta_{4}$ & $\eta_{5}$ \\
\hline 1 & 0.07938 & 0 & 0.1057 & 0.01754 & -0.08815 \\
\hline 2 & 0.04132 & 0 & 0.05518 & 0.009236 & -0.04594 \\
\hline 3 & 0.02792 & 0 & 0.03731 & 0.006258 & -0.03105 \\
\hline 4 & 0.02108 & 0 & 0.02818 & 0.004731 & -0.02345 \\
\hline 5 & 0.01693 & 0 & 0.02264 & 0.003802 & -0.01883 \\
\hline 6 & 0.01415 & 0 & 0.01892 & 0.003178 & -0.01574 \\
\hline 7 & 0.01215 & 0 & 0.01625 & 0.00273 & -0.01352 \\
\hline 8 & 0.01065 & 0 & 0.01424 & 0.002393 & -0.01184 \\
\hline 9 & 0.009474 & 0 & 0.01267 & 0.00213 & -0.01054 \\
\hline 10 & 0.008534 & 0 & 0.01141 & 0.001919 & -0.009494 \\
\hline \hline
\end{tabular}

\section{Diamagnetic Susceptibilities}

In the free fermion system, the diamagnetic susceptibilities within the $x$-y plane and along $z$-axis satisfy

$$
\Delta \chi_{D}^{\perp} \sim \sqrt{A} e^{2} \sqrt{T} \quad \text { and } \quad \Delta \chi_{D}^{z} \sim \frac{B^{2 / 3}}{\sqrt{A}} e^{2} T^{5 / 6} .
$$

Here, $\Delta \chi_{D}^{\perp}=\chi_{D}^{\perp}-\chi_{D 0}^{\perp}$ and $\Delta \chi_{D}^{z}=\chi_{D}^{z}-\chi_{D 0}^{z}$, where $\chi_{D}^{\perp}$ and $\chi_{D 0}^{z}$ are the residual constant value at $T=0$. Considering the influence of Coulomb interaction, $\Delta \chi_{D}^{\perp}$ and $\Delta \chi_{D}^{z}$ become

$$
\begin{aligned}
& \frac{d \ln \left(\Delta \chi_{D}^{\perp}\right)}{d \ln (T)} \sim \frac{1}{2}+\frac{d \ln \left(\sqrt{A} e^{2}\right)}{d \ln (T)} \\
& \frac{d \ln \left(\Delta \chi_{D}^{z}\right)}{d \ln (T)} \sim \frac{5}{6}+\frac{d \ln \left(\frac{B^{2 / 3}}{\sqrt{A}} e^{2}\right)}{d \ln (T)} .
\end{aligned}
$$

Employing the transformation $T=T_{0} e^{-\ell}$, we get

$$
\frac{d \ln (e)}{d \ln (T)}=-\left(\frac{1}{4}-\frac{N C_{\perp}}{2}\right) \text {. }
$$

Substituting Eqs. (E13), (E14), and (E35) into Eqs. (E33) and (E34), it is easy to find that

$$
\begin{aligned}
\frac{d \ln \left(\Delta \chi_{D}^{\perp}\right)}{d \ln (T)} & \sim \frac{1}{2}-\frac{1}{2}+N C_{\perp}-\frac{1}{2} C_{3} \\
& \sim \frac{1}{2}-\frac{1}{2}+N C_{\perp}^{*}-\frac{1}{2} C_{3}^{*} \\
\frac{d \ln \left(\Delta \chi_{D}^{z}\right)}{d \ln (T)} & \sim \frac{5}{6}-\frac{1}{2}+N C_{\perp}-\frac{2}{3} C_{2}+\frac{1}{2} C_{3} \\
& \sim \frac{5}{6}-\frac{1}{2}+N C_{\perp}^{*}-\frac{2}{3} C_{2}^{*}+\frac{1}{2} C_{3}^{*},
\end{aligned}
$$

which directly give rise to the following renormalized dynamical susceptibilities

$$
\begin{aligned}
& \Delta \chi_{D}^{\perp}(T) \sim T^{\frac{1}{2}-\frac{1}{2}+N C_{\perp}^{*}-\frac{1}{2} C_{3}^{*}} \sim T^{1 / 2+\eta_{4}} \\
& \Delta \chi_{D}^{z}(T) \sim T^{\frac{5}{6}-\frac{1}{2}+N C_{\perp}^{*}-\frac{2}{3} C_{2}^{*}+\frac{1}{2} C_{3}^{*}} \sim T^{5 / 6+\eta_{5}}
\end{aligned}
$$

where

$$
\begin{aligned}
& \eta_{4}=-\frac{1}{2}+N C_{\perp}^{*}-\frac{1}{2} C_{3}^{*}, \\
& \eta_{5}=-\frac{1}{2}+N C_{\perp}^{*}-\frac{2}{3} C_{2}^{*}+\frac{1}{2} C_{3}^{*} .
\end{aligned}
$$

The values of $\eta_{1}, \eta_{2}, \eta_{3}, \eta_{4}$, and $\eta_{5}$ for a series of flavors are given in Table III

\section{Appendix F: Interplay between long-range and short-range interactions}

Here, we study the interplay between the Coulomb interaction and some types of four-fermion interactions, and analyze the nontrivial physical effects of such an interplay. 


\section{Fierz identity}

The four-fermion-type short-range interactions between fermions can be generically written as

$$
\begin{aligned}
S_{\psi^{4}}= & \frac{1}{N} \int d \tau d^{3} \mathbf{x}\left[\lambda_{0}\left(\psi^{\dagger} \sigma_{0} \psi\right)^{2}+\lambda_{1}\left(\psi^{\dagger} \sigma_{1} \psi\right)^{2}\right. \\
& \left.+\lambda_{2}\left(\psi^{\dagger} \sigma_{2} \psi\right)^{2}+\lambda_{3}\left(\psi^{\dagger} \sigma_{3} \psi\right)^{2}\right]
\end{aligned}
$$

where $\sigma_{0}$ is the identity matrix. As dictated by the Fierz identity, the four coupling terms $\left(\psi^{\dagger} \sigma_{0} \psi\right)^{2} \equiv\left(\psi^{\dagger} \psi\right)^{2}$, $\left(\psi^{\dagger} \sigma_{1} \psi\right)^{2},\left(\psi^{\dagger} \sigma_{2} \psi\right)^{2},\left(\psi^{\dagger} \sigma_{3} \psi\right)^{2}$ are not independent [28, 49. According to the Fierz identity, one can find that [28, 49.

$$
\left[\psi^{\dagger}(x) \sigma_{a} \psi(x)\right]\left[\psi^{\dagger}(y) \sigma_{b} \psi(y)\right]=-\frac{1}{4} \operatorname{Tr}\left[\sigma_{a} \sigma_{c} \sigma_{b} \sigma_{d}\right]\left[\psi^{\dagger}(x) \sigma_{c} \psi(y)\right]\left[\psi^{\dagger}(y) \sigma_{d} \psi(x)\right],
$$

where $a, b, c, d=0,1,2,3$. Locality requires that $x=y$, which leads to

$$
\left[\psi^{\dagger}(x) \sigma_{a} \psi(x)\right]\left[\psi^{\dagger}(x) \sigma_{b} \psi(x)\right]=-\frac{1}{4} \operatorname{Tr}\left[\sigma_{a} \sigma_{c} \sigma_{b} \sigma_{d}\right]\left[\psi^{\dagger}(x) \sigma_{c} \psi(x)\right]\left[\psi^{\dagger}(x) \sigma_{d} \psi(x)\right] .
$$

From this equation, we get

$$
\begin{aligned}
& 3\left[\psi^{\dagger}(x) \sigma_{0} \psi(x)\right]^{2}+\left[\psi^{\dagger}(x) \sigma_{1} \psi(x)\right]^{2}+\left[\psi^{\dagger}(x) \sigma_{2} \psi(x)\right]^{2}+\left[\psi^{\dagger}(x) \sigma_{3} \psi(x)\right]^{2}=0 \\
& {\left[\psi^{\dagger}(x) \sigma_{0} \psi(x)\right]^{2}+3\left[\psi^{\dagger}(x) \sigma_{1} \psi(x)\right]^{2}-\left[\psi^{\dagger}(x) \sigma_{2} \psi(x)\right]^{2}-\left[\psi^{\dagger}(x) \sigma_{3} \psi(x)\right]^{2}=0} \\
& {\left[\psi^{\dagger}(x) \sigma_{0} \psi(x)\right]^{2}-\left[\psi^{\dagger}(x) \sigma_{1} \psi(x)\right]^{2}+3\left[\psi^{\dagger}(x) \sigma_{2} \psi(x)\right]^{2}-\left[\psi^{\dagger}(x) \sigma_{3} \psi(x)\right]^{2}=0,} \\
& {\left[\psi^{\dagger}(x) \sigma_{0} \psi(x)\right]^{2}-\left[\psi^{\dagger}(x) \sigma_{1} \psi(x)\right]^{2}-\left[\psi^{\dagger}(x) \sigma_{2} \psi(x)\right]^{2}+3\left[\psi^{\dagger}(x) \sigma_{3} \psi(x)\right]^{2}=0 .}
\end{aligned}
$$

Defining

$$
X=\left(\begin{array}{c}
\left(\psi^{\dagger} \sigma_{0} \psi\right)^{2} \\
\left(\psi^{\dagger} \sigma_{1} \psi\right)^{2} \\
\left(\psi^{\dagger} \sigma_{2} \psi\right)^{2} \\
\left(\psi^{\dagger} \sigma_{3} \psi\right)^{2}
\end{array}\right)
$$

and

$$
F=\left(\begin{array}{cccc}
3 & 1 & 1 & 1 \\
1 & 3 & -1 & -1 \\
1 & -1 & 3 & -1 \\
1 & -1 & 3 & -1
\end{array}\right)
$$

we now can re-write Eqs. (F4)-( $\mathrm{F} 7 \mathrm{l}$ ) in a more compact form

$$
F X=0
$$

Solving this equation gives rise to

$$
-\left(\psi^{\dagger} \sigma_{0} \psi\right)^{2}=\left(\psi^{\dagger} \sigma_{1} \psi\right)^{2}=\left(\psi^{\dagger} \sigma_{2} \psi\right)^{2}=\left(\psi^{\dagger} \sigma_{3} \psi\right)^{2}
$$

Similar to previous works on four-fermion interactions [28, 49, 57], we will use Eq. (F11) to carry out RG calculations.

\section{Physical meaning of fermion bilinears}

Upon decoupling the four-fermion interaction terms and then taking the expectation value, we obtain four different fermion bilinears:

$$
\begin{aligned}
& \Delta_{0} \equiv\left\langle\psi^{\dagger} \sigma_{0} \psi\right\rangle \equiv\left\langle\psi^{\dagger} \psi\right\rangle, \quad \Delta_{1} \equiv\left\langle\psi^{\dagger} \sigma_{1} \psi\right\rangle, \\
& \Delta_{2} \equiv\left\langle\psi^{\dagger} \sigma_{2} \psi\right\rangle, \quad \Delta_{3} \equiv\left\langle\psi^{\dagger} \sigma_{3} \psi\right\rangle .
\end{aligned}
$$

When the coupling parameter $\lambda_{i}$, where $i=0,1,2,3$, diverges at low energies, the corresponding $\Delta_{i}$ acquires a finite value. Each fermion billinear has its special physical meaning. At the TQCP, the original fermion dispersion is given by

$$
E= \pm \sqrt{B^{2} d_{1}^{2}(\mathbf{k})+B^{2} d_{2}^{2}(\mathbf{k})+A^{2} d_{3}^{2}(\mathbf{k})}
$$

which vanishes at the point $(0,0,0)$. This dispersion will be altered if $\Delta_{i}$ becomes finite.

If $\Delta_{0} \equiv\left\langle\psi^{\dagger} \sigma_{0} \psi\right\rangle$ acquires a finite value, the fermion dispersion becomes

$$
E=\Delta_{0} \pm \sqrt{B^{2} d_{1}^{2}(\mathbf{k})+B^{2} d_{2}^{2}(\mathbf{k})+A^{2} d_{3}^{2}(\mathbf{k})}
$$

which vanishes not at $(0,0,0)$ but on the surface $\sqrt{B^{2} d_{1}^{2}(\mathbf{k})+B^{2} d_{2}^{2}(\mathbf{k})+A^{2} d_{3}^{2}(\mathbf{k})}=\left|\Delta_{0}\right|$. It is easy to see that $\Delta_{0} \equiv\left\langle\psi^{\dagger} \sigma_{0} \psi\right\rangle$ represents finite chemical potential. 
If $\Delta_{1} \equiv\left\langle\psi^{\dagger} \sigma_{1} \psi\right\rangle$ becomes finite, the fermion dispersion is changed into

$$
E= \pm \sqrt{\left(B d_{1}(\mathbf{k})+\Delta_{1}\right)^{2}+B^{2} d_{2}^{2}(\mathbf{k})+A^{2} d_{3}^{2}(\mathbf{k})}
$$

This dispersion vanishes at three different points:

$$
\begin{aligned}
& \left\{\begin{array}{l}
k_{a x}=-\left(\frac{\Delta_{1}}{B}\right)^{1 / 3} \\
k_{a y}=0 \\
k_{a z}=0
\end{array}\right. \\
& \left\{\begin{array}{l}
k_{c x}=\frac{1}{2}\left(\frac{\Delta_{1}}{B}\right)^{1 / 3} \\
k_{c y}=-\frac{\sqrt{3}}{2}\left(\frac{\Delta_{1}}{B}\right)^{1 / 3} . \\
k_{c z}=0
\end{array}\right.
\end{aligned}
$$

One can expand the dispersion in the vicinity of these three points. We define $\mathbf{K}$ as the momentum relative to the gapless points, and then find that

$$
E= \pm \sqrt{9 B^{2 / 3} \Delta_{1}^{4 / 3} K_{\perp}^{2}+A^{2} K_{z}^{4}}
$$

which is linear along two directions and quadratic along the third one. Notice that the above effective dispersion is the same for all these three gapless points. One can identify $\Delta_{1}$ as a nematic order parameter, because the positions of these three points break the equivalence between $x$ - and $y$-axis.

If $\Delta_{2}=\left\langle\psi^{\dagger} \sigma_{2} \psi\right\rangle$ becomes finite, the fermion dispersion has the new form

$$
E= \pm \sqrt{B d_{1}^{2}(\mathbf{k})+\left(B d_{2}(\mathbf{k})+\Delta_{2}\right)^{2}+A^{2} d_{3}^{2}(\mathbf{k})}
$$

This energy also vanishes at three gapless points

$$
\begin{aligned}
& \left\{\begin{array}{l}
k_{a x}=0 \\
k_{a y}=-\left(\frac{\Delta_{2}}{B}\right)^{1 / 3} \\
k_{a z}=0
\end{array}, \quad\left\{\begin{array}{l}
k_{b x}=\frac{\sqrt{3}}{2}\left(\frac{\Delta_{2}}{B}\right)^{1 / 3} \\
k_{b y}=\frac{1}{2}\left(\frac{\Delta_{2}}{B}\right)^{1 / 3} \\
k_{b z}=0
\end{array}\right.\right. \\
& \left\{\begin{array}{l}
k_{c x}=-\frac{\sqrt{3}}{2}\left(\frac{\Delta_{2}}{B}\right)^{1 / 3} \\
k_{c y}=\frac{1}{2}\left(\frac{\Delta_{2}}{B}\right)^{1 / 3} \\
k_{c z}=0
\end{array}\right.
\end{aligned}
$$

Introducing $\mathbf{K}$ as the momentum relative to gapless points, the effective dispersion becomes

$$
E \pm \sqrt{9 B^{2 / 3} \Delta_{2}^{4 / 3} K_{\perp}^{2}+A^{2} K_{z}^{4}}
$$

which is also linear along two directions and quadratic along the third. Apparently, the impact of finite $\Delta_{2}$ is qualitatively very similar to that of finite $\Delta_{1}$. Thus, $\Delta_{2}$ can also be identified as a nematic order parameter.

If $\Delta_{3}=\left\langle\psi^{\dagger} \sigma_{3} \psi\right\rangle$ becomes finite. the fermion dispersion reads

$$
E= \pm \sqrt{B^{2} d_{1}^{2}(\mathbf{k})+B^{2} d_{2}^{2}(\mathbf{k})+\left(A d_{3}(\mathbf{k})+\Delta_{3}\right)^{2}} .
$$

The energy gap for band insultor vanishes continuously upon approaching the TQCP when $\Delta_{3}=0$. In contrast, this gap vanishes abruptly at the TQCP once $\Delta_{3} \neq 0$. Therefore, the TQPT between triple-WSM and BI becomes first order if $\left\langle\psi^{\dagger} \sigma_{3} \psi\right\rangle \neq 0$. Recently, Roy et al. [49] have studied the four-fermion interaction $\left(\psi^{\dagger} \sigma_{3} \psi\right)^{2}$ at the TQCP between triple-WSM and BI. This interaction is found to be irrelevant if the initial strength is small. If the initial strength is larger than some critical value, the mean value $\left\langle\psi^{\dagger} \sigma_{3} \psi\right\rangle$ becomes finite and the original continuous TQPT becomes first order [49].

Below we will combine the Coulomb interaction with every possible four-fermion interaction. The interplay between Coulomb interaction and $\left(\psi^{\dagger} \sigma_{1} \psi\right)^{2}$ leads to qualitatively the same low-energy properties as that between Coulomb interaction and $\left(\psi^{\dagger} \sigma_{2} \psi\right)^{2}$. Therefore, we only need to consider the former case.

\section{Interplay with $\left(\psi^{\dagger} \sigma_{1} \psi\right)^{2}$}

We will derive the flow equation for the coupling parameter $\lambda_{1}$. For this purpose, we need to compute the Feynman diagrams for the vertex corrections to $\left(\psi^{\dagger} \sigma_{1} \psi\right)^{2}$. All the involved diagrams are presented in Fig. 6.

The contribution from the diagram of Fig. 6(a) is given by

$$
\begin{aligned}
\delta \lambda_{1}^{(1)} \sigma_{1}= & \frac{4 \lambda_{1}^{2}}{N} \int^{\prime} \frac{d \omega}{2 \pi} \frac{d^{3} \mathbf{k}}{(2 \pi)^{3}} \sigma_{1} G_{0}(i \omega, \mathbf{k}) \sigma_{1} G_{0}(i \omega, \mathbf{k}) \\
& \times \sigma_{1} .
\end{aligned}
$$

Substituting Eq. (A2) into Eq. (F22) and then employing the transformations Eqs. (A9)-(A11), we obtain

$$
\delta \lambda_{1}^{(1)}=-\frac{\lambda_{1}^{2}}{N} \frac{5 \Gamma\left(\frac{5}{4}\right) \Gamma\left(\frac{4}{3}\right) \Lambda^{1 / 6}}{12 \pi^{2} \Gamma\left(\frac{19}{12}\right) \sqrt{A} B^{2 / 3}} \ell .
$$

Diagrams shown in Figs. 6(b) and 6(c) give rise to the correction

$$
\begin{aligned}
V_{1}^{(2)+(3)}= & \frac{4 \lambda_{1}^{2}}{N} \int^{\prime} \frac{d \omega}{2 \pi} \frac{d^{3} \mathbf{k}}{(2 \pi)^{3}}\left(\psi^{\dagger} \sigma_{1} G_{0}(i \omega, \mathbf{k}) \sigma_{1} \psi\right) \\
& \times\left\{\psi ^ { \dagger } \left[\sigma_{1} G_{0}(i \omega, \mathbf{k}) \sigma_{1}\right.\right. \\
& \left.\left.+\sigma_{1} G_{0}(-i \omega,-\mathbf{k}) \sigma_{1}\right] \psi\right\} \\
= & \frac{\lambda_{1}^{2}}{N} \frac{\Gamma\left(\frac{1}{3}\right) \Gamma\left(\frac{5}{4}\right) \Lambda^{1 / 6}}{12 \pi^{2} \Gamma\left(\frac{19}{12}\right) \sqrt{A} B^{2 / 3}} \ell\left(\psi^{\dagger} \sigma_{3} \psi\right)^{2} .(\mathrm{F} 24)
\end{aligned}
$$

Making use of Fierz identity, we further have

$$
V_{1}^{(2)+(3)}=\frac{\lambda_{1}^{2}}{N} \frac{\Gamma\left(\frac{1}{3}\right) \Gamma\left(\frac{5}{4}\right) \Lambda^{1 / 6}}{12 \pi^{2} \Gamma\left(\frac{19}{12}\right) \sqrt{A} B^{2 / 3}} \ell\left(\psi^{\dagger} \sigma_{1} \psi\right)^{2} .
$$

Similarly, we get

$$
\delta \lambda_{1}^{(2)+(3)}=\frac{\lambda_{1}^{2}}{N} \frac{\Gamma\left(\frac{1}{3}\right) \Gamma\left(\frac{5}{4}\right) \Lambda^{1 / 6}}{12 \pi^{2} \Gamma\left(\frac{19}{12}\right) \sqrt{A} B^{2 / 3}} \ell .
$$



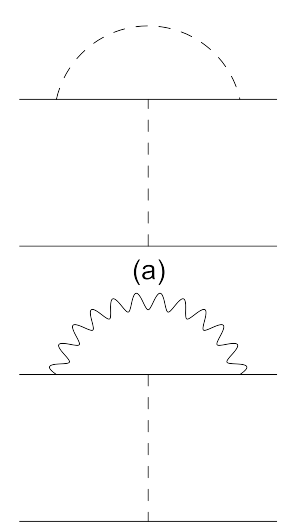

(e)

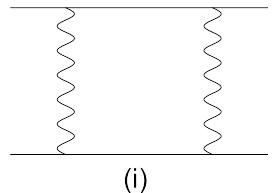

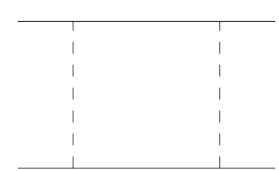

(b)

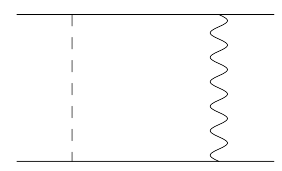

(f)

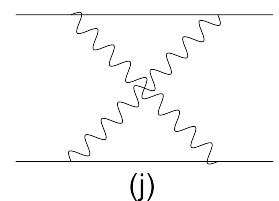

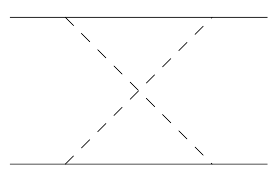

(c)

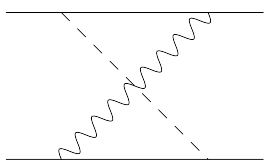

(g)

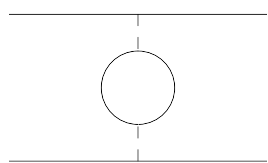

(d)

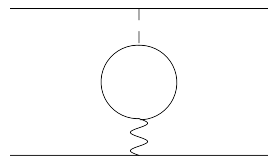

(h)

FIG. 6: Feynman diagrams for the vertex corrections to four-fermion interaction. Solid, wavy, and dashed lines represent the fermion propagator, Coulomb interaction, and four-fermion interaction, respectively.

The correction of diagram Fig. 6(d) is given by

$$
\begin{aligned}
\delta \lambda_{1}^{(4)}= & -2 N \frac{\lambda_{1}^{2}}{N} \int^{\prime} \frac{d \omega}{2 \pi} \frac{d^{3} \mathbf{k}}{(2 \pi)^{3}} \operatorname{Tr}\left[\sigma_{1} G_{0}(i \omega, \mathbf{k}) \sigma_{1}\right. \\
& \left.\times G_{0}(i \omega, \mathbf{k})\right] \\
= & \lambda_{1}^{2} \frac{5 \Gamma\left(\frac{5}{4}\right) \Gamma\left(\frac{4}{3}\right) \Lambda^{1 / 6}}{12 \pi^{2} \Gamma\left(\frac{19}{12}\right) \sqrt{A} B^{2 / 3}} \ell .
\end{aligned}
$$

The diagram shown in Fig. 6(e) yields

$$
\begin{aligned}
\delta \lambda_{1}^{(5)} \sigma_{1}= & -2 g^{2} \lambda_{1} \int^{\prime} \frac{d \Omega}{2 \pi} \frac{d^{3} \mathbf{q}}{(2 \pi)^{3}} G_{0}(i \Omega, \mathbf{q}) \sigma_{1} G_{0}(i \Omega, \mathbf{q}) \\
& \times D_{0}(i \Omega, \mathbf{q}) .
\end{aligned}
$$

Carrying out direct calculations, we obtain

$$
\delta \lambda_{1}^{(5)}=\lambda_{1} C_{4} \ell
$$

where

$$
\begin{aligned}
C_{4}= & \frac{g^{2}}{24 \pi^{2} \sqrt{A \Lambda}} \int_{0}^{+\infty} d \xi \frac{2+\xi^{2}}{\xi^{1 / 3}\left(1+\xi^{2}\right)^{13 / 12}} \\
& \times \frac{1}{\xi^{2 / 3}\left(1+\xi^{2}\right)^{1 / 6}+\zeta}
\end{aligned}
$$

For diagrams given by Figs. 6(f) and 6(g), we get

$$
\begin{aligned}
V_{1}^{(6)+(7)}= & -4 g^{2} \lambda_{1} \int^{\prime} \frac{d \Omega}{2 \pi} \frac{d^{3} \mathbf{q}}{(2 \pi)^{3}}\left(\psi_{\sigma}^{\dagger} \sigma_{1} G_{0}(i \Omega, \mathbf{q}) \psi_{\sigma}\right) \\
& \times\left\{\psi^{\dagger}\left[G_{0}(i \Omega, \mathbf{q}) \sigma_{1}+\sigma_{1} G_{0}(-i \Omega,-\mathbf{q})\right] \psi\right\} \\
& \times D_{0}(i \Omega, \mathbf{q}) \\
\approx & \lambda_{1} C_{5} \ell\left(\psi^{\dagger} \sigma_{3} \psi\right)^{2},
\end{aligned}
$$

where

$$
\begin{aligned}
C_{5}= & -\frac{g^{2}}{12 \pi^{2} \sqrt{A \Lambda}} \int_{0}^{+\infty} d \xi \frac{\xi^{5 / 3}}{\left(1+\xi^{2}\right)^{13 / 12}} \\
& \times \frac{1}{\xi^{2 / 3}\left(1+\xi^{2}\right)^{1 / 6}+\zeta} .
\end{aligned}
$$

Fierz identity allows us to recast Eq. F31 in the form

$$
V_{1}^{(6)+(7)}=\lambda_{1} C_{5} \ell\left(\psi^{\dagger} \sigma_{1} \psi\right)^{2},
$$

which indicates that

$$
\delta \lambda_{1}^{(6)+(7)}=\lambda_{1} C_{5} \ell .
$$

The correction due to the diagram shown in Fig. 6(h) is found to vanish, namely

$$
\begin{aligned}
\delta \lambda_{1}^{(8)}= & 2 \lambda_{1} g^{2} \int^{\prime} \frac{d \omega}{2 \pi} \frac{d^{3} \mathbf{k}}{(2 \pi)^{3}} \operatorname{Tr}\left[G_{0}(i \omega, \mathbf{k}) \sigma_{1}\right. \\
& \left.\times G_{0}(i \omega+i \Omega, \mathbf{k}+\mathbf{q})\right] D_{0}(i \Omega, \mathbf{q}) \\
= & 0 .
\end{aligned}
$$

Figs. 6(i) and 6(j) induce the contribution

$$
\begin{aligned}
V_{1}^{(9)+(10)}= & 4 g^{4} \int^{\prime} \frac{d \Omega}{2 \pi} \frac{d^{3} \mathbf{q}}{(2 \pi)^{3}}\left(\psi^{\dagger} G_{0}(i \Omega, \mathbf{q}) \psi\right) D_{0}(i \Omega, \mathbf{q}) \\
& \times\left\{\psi^{\dagger}\left[G_{0}(i \Omega, \mathbf{q})+G_{0}(-i \Omega,-\mathbf{q})\right] \psi\right\} \\
& \times D_{0}(i \Omega, \mathbf{q}) \\
= & \frac{\sqrt{A} B^{2 / 3}}{\Lambda^{1 / 6}} C_{6} \ell\left(\psi^{\dagger} \sigma_{3} \psi\right)^{2}, \quad(\mathrm{~F} 36)
\end{aligned}
$$


where

$$
\begin{aligned}
C_{6}= & \frac{g^{4}}{6 \pi^{2} A \Lambda} \int_{0}^{+\infty} d \xi \frac{1}{\xi^{1 / 3}\left(1+\xi^{2}\right)^{7 / 12}} \\
& \times \frac{1}{\left[\xi^{2 / 3}\left(1+\xi^{2}\right)^{1 / 6}+\zeta\right]^{2}} .
\end{aligned}
$$

We learn from Fierz identity that Eq. (F36) can also be written as

$$
V_{1}^{(9)+(10)}=\frac{\sqrt{A} B^{2 / 3}}{\Lambda^{1 / 6}} C_{6} \ell\left(\psi^{\dagger} \sigma_{1} \psi\right)^{2},
$$

which gives rise to

$$
\delta \lambda_{1}^{(9)+(10)}=\frac{\sqrt{A} B^{2 / 3}}{\Lambda^{1 / 6}} C_{6} \ell .
$$

The total corrections to coupling parameter $\lambda_{1}$ are

$$
\begin{aligned}
\delta \lambda_{1}= & \delta \lambda_{1}^{(1)}+\delta \lambda_{1}^{(2)+(3)}+\delta \lambda_{1}^{(4)}+\delta \lambda_{1}^{(5)}+\delta \lambda_{1}^{(6)+(7)} \\
& +\delta \lambda_{1}^{(8)}+\delta \lambda_{1}^{(9)+(10)} \\
= & \left(1-\frac{2}{5 N}\right) \lambda_{1}^{2} \frac{5 \Gamma\left(\frac{5}{4}\right) \Gamma\left(\frac{4}{3}\right) \Lambda^{1 / 6}}{12 \pi^{2} \Gamma\left(\frac{19}{12}\right) \sqrt{A} B^{2 / 3}} \ell \\
& +\lambda_{1}\left(C_{4}+C_{5}\right) \ell+\frac{\sqrt{A} B^{2 / 3}}{\Lambda^{1 / 6}} C_{6} \ell .
\end{aligned}
$$

Including these corrections to the original action leads to

$$
\begin{aligned}
S_{\psi^{4}}= & \frac{1}{N}\left(\lambda_{1}+\delta \lambda_{1}\right) \int \frac{d \omega_{1}}{2 \pi} \frac{d^{3} \mathbf{k}_{1}}{(2 \pi)^{3}} \frac{d \omega_{2}}{2 \pi} \frac{d^{3} \mathbf{k}_{2}}{(2 \pi)^{3}} \frac{d \omega_{3}}{2 \pi} \frac{d^{3} \mathbf{k}_{3}}{(2 \pi)^{3}} \\
& \times \psi_{a}^{\dagger}\left(\omega_{1}, \mathbf{k}_{1}\right) \sigma_{1} \psi_{a}\left(\omega_{2}, \mathbf{k}_{2}\right) \psi_{a}^{\dagger}\left(\omega_{3}, k_{3}\right) \sigma_{1} \\
& \times \psi_{a}\left(\omega_{1}-\omega_{2}+\omega_{3}, \mathbf{k}_{1}-\mathbf{k}_{2}+\mathbf{k}_{3}\right) .
\end{aligned}
$$

Employing the transformations Eqs. (C3)-(C7), we convert it into

$$
\begin{aligned}
S_{\psi^{\prime 4}} \approx & \frac{1}{N}\left(\lambda_{1}-\lambda_{1} \frac{\ell}{6}+\delta \lambda_{1}\right) \int \frac{d \omega_{1}^{\prime}}{2 \pi} \frac{d^{3} \mathbf{k}_{1}^{\prime}}{(2 \pi)^{3}} \frac{d \omega_{2}^{\prime}}{2 \pi} \frac{d^{3} \mathbf{k}_{2}^{\prime}}{(2 \pi)^{3}} \\
& \times \frac{d \omega_{3}^{\prime}}{2 \pi} \frac{d^{3} \mathbf{k}_{3}^{\prime}}{(2 \pi)^{3}} \psi_{a}^{\prime \dagger}\left(\omega_{1}^{\prime}, \mathbf{k}_{1}^{\prime}\right) \sigma_{1} \psi_{a}^{\prime}\left(\omega_{2}^{\prime}, \mathbf{k}_{2}^{\prime}\right) \psi_{a}^{\prime \dagger}\left(\omega_{3}^{\prime}, k_{3}^{\prime}\right) \\
& \times \sigma_{1} \psi_{a}^{\prime}\left(\omega_{1}^{\prime}-\omega_{2}^{\prime}+\omega_{3}^{\prime}, \mathbf{k}_{1}^{\prime}-\mathbf{k}_{2}^{\prime}+\mathbf{k}_{3}^{\prime}\right) . \quad(\mathrm{F} 42
\end{aligned}
$$

By introducing a renormalized coupling parameter

$$
\lambda_{1}^{\prime}=\lambda_{1}-\lambda_{1} \frac{\ell}{6}+\delta \lambda_{1},
$$

the original form of the action can be recovered. From Eqs. (F40) and (F43), we obtain the following flow equation for $\lambda_{1}$ :

$$
\begin{aligned}
\frac{d \lambda_{1}}{d \ell}= & -\frac{1}{6} \lambda_{1}+\left(1-\frac{2}{5 N}\right) \lambda_{1}^{2}+\left(C_{4}+C_{5}-\frac{1}{2} C_{3}\right. \\
& \left.-\frac{2}{3} C_{2}\right) \lambda_{1}+C_{7},
\end{aligned}
$$

where

$$
C_{7}=\frac{5 \Gamma\left(\frac{5}{4}\right) \Gamma\left(\frac{4}{3}\right)}{12 \pi^{2} \Gamma\left(\frac{19}{12}\right)} C_{6} .
$$

In the derivation of the above equation, we have made the redefinition

$$
\frac{5 \Gamma\left(\frac{5}{4}\right) \Gamma\left(\frac{4}{3}\right) \Lambda^{1 / 6}}{12 \pi^{2} \Gamma\left(\frac{19}{12}\right) \sqrt{A} B^{2 / 3}} \lambda_{1} \rightarrow \lambda_{1}
$$

In Figs. 7(a)-7(d), we show the RG flow of $\lambda_{1}$ due to the interplay of four-fermion interaction $\left(\psi^{\dagger} \sigma_{1} \psi\right)^{2}$ and Coulomb interaction. The corresponding flow diagrams on the $\alpha-\lambda_{1}$ plane are displayed in Figs. $7(\mathrm{e})-7(\mathrm{~h})$. We observe that, $\lambda_{1}$ increases quickly from zero as $\ell$ grows and goes to infinity at a finite value $\ell_{c}$. The fermion bilinear $\psi^{\dagger} \sigma_{1} \psi$ acquires a finite expectation value, i.e., $\left\langle\psi^{\dagger} \sigma_{1} \psi\right\rangle \neq 0$. Now a nematic order develops in the system, and splits the original band-touching point into three different touching points. Around the new points, the fermion dispersion is linear along two out of three directions and quadratic along the rest one.

\section{Interplay with $\left(\psi^{\dagger} \sigma_{3} \psi\right)^{2}$}

The correction from Fig. 6](a) is given by

$$
\begin{aligned}
\delta \lambda_{3}^{(1)} \sigma_{3}= & \frac{4 \lambda_{3}^{2}}{N} \int^{\prime} \frac{d \omega}{2 \pi} \frac{d^{3} \mathbf{k}}{(2 \pi)^{3}} \sigma_{3} G_{0}(i \omega, \mathbf{k}) \sigma_{3} \\
& \times G_{0}(i \omega, \mathbf{k}) \sigma_{3} .
\end{aligned}
$$

Substituting Eq. (A2) into Eq. (F47) and carrying direct calculations, we find

$$
\delta \lambda_{3}^{(1)}=-\frac{\lambda_{3}^{2}}{N} \frac{\Gamma\left(\frac{5}{4}\right) \Gamma\left(\frac{4}{3}\right) \Lambda^{1 / 6}}{3 \pi^{2} \Gamma\left(\frac{19}{12}\right) \sqrt{A} B^{2 / 3}} \ell .
$$

The contribution induced by Figs. 6(b) and 6(c) can be written as

$$
\begin{aligned}
V_{3}^{(2)+(3)}= & \frac{4 \lambda_{3}^{2}}{N} \int^{\prime} \frac{d \omega}{2 \pi} \frac{d^{3} \mathbf{k}}{(2 \pi)^{3}}\left(\psi^{\dagger} \sigma_{3} G_{0}(i \omega, \mathbf{k}) \sigma_{3} \psi\right) \\
& \times\left\{\psi ^ { \dagger } \left[\sigma_{3} G_{0}(i \omega, \mathbf{k}) \sigma_{3}\right.\right. \\
& \left.\left.+\sigma_{3} G_{0}(-i \omega,-\mathbf{k}) \sigma_{3}\right] \psi\right\} \\
= & \frac{\lambda_{3}^{2}}{N} \frac{\Gamma\left(\frac{1}{3}\right) \Gamma\left(\frac{5}{4}\right) \Lambda^{1 / 6}}{12 \pi^{2} \Gamma\left(\frac{19}{12}\right) \sqrt{A} B^{2 / 3}} \ell\left(\psi^{\dagger} \sigma_{3} \psi\right)^{2},(\mathrm{~F} 49)
\end{aligned}
$$

which represents

$$
\delta \lambda_{3}^{(2)+(3)}=\frac{\lambda_{3}^{2}}{N} \frac{\Gamma\left(\frac{1}{3}\right) \Gamma\left(\frac{5}{4}\right) \Lambda^{1 / 6}}{12 \pi^{2} \Gamma\left(\frac{19}{12}\right) \sqrt{A} B^{2 / 3}} \ell .
$$

Fig. 6(d) results in the correction

$$
\begin{aligned}
\delta \lambda_{3}^{(4)}= & -2 N \frac{\lambda_{3}^{2}}{N} \int^{\prime} \frac{d \omega}{2 \pi} \frac{d^{3} \mathbf{k}}{(2 \pi)^{3}} \operatorname{Tr}\left[\sigma_{3} G_{0}(i \omega, \mathbf{k}) \sigma_{3}\right. \\
& \left.\times G_{0}(i \omega, \mathbf{k})\right] \\
= & \lambda_{3}^{2} \frac{\Gamma\left(\frac{5}{4}\right) \Gamma\left(\frac{4}{3}\right) \Lambda^{1 / 6}}{3 \pi^{2} \Gamma\left(\frac{19}{12}\right) \sqrt{A} B^{2 / 3}} \ell .
\end{aligned}
$$



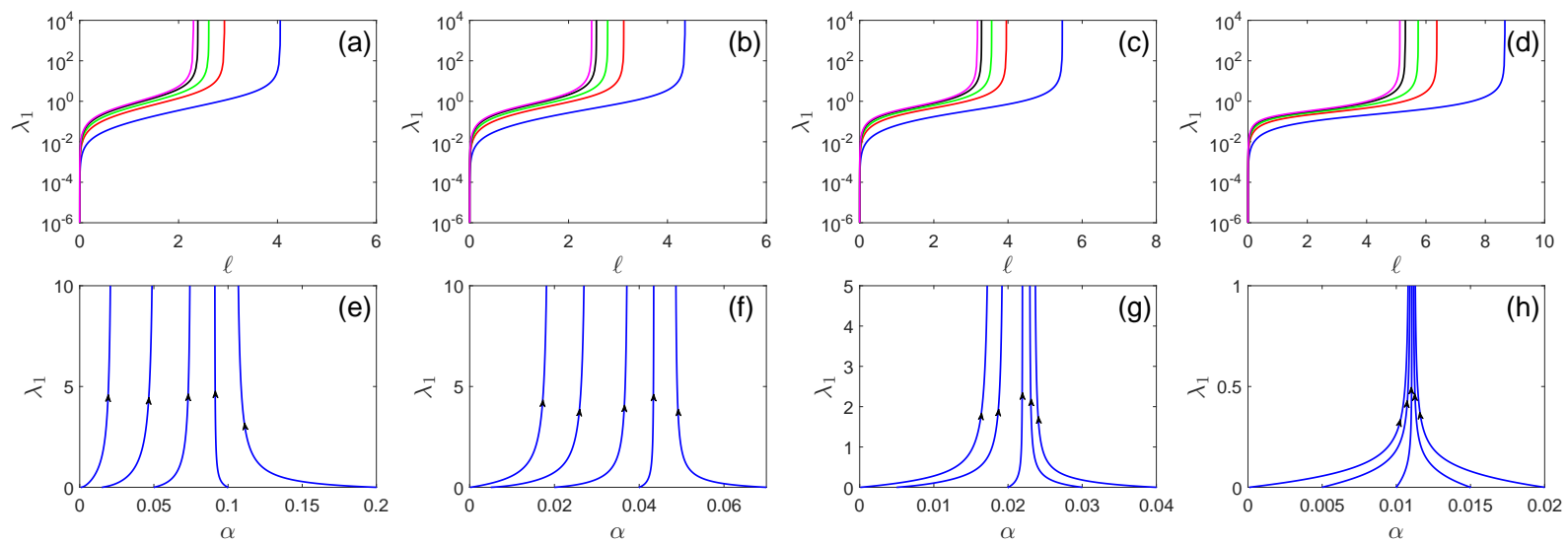

FIG. 7: (a)-(d): Dependence of $\lambda_{1}$ on $\ell$ at $N=1, N=2, N=4, N=8$, respectively. Blue, red, green, black, magenta lines correspond to $\alpha_{0}=0.01,0.05,0.1,0.2,0.3$, respectively. (e)-(h): Flow diagrams on the $\alpha-\lambda_{1}$ plane at $N=1, N=2, N=4$, and $N=8$, respectively. Here, we take $\beta_{0}=0.1$.

The correction induced by Fig. [6) (e) reads as

$$
\begin{aligned}
\delta \lambda_{3}^{(5)} \sigma_{3}= & -2 g^{2} \lambda_{3} \int^{\prime} \frac{d \Omega}{2 \pi} \frac{d^{3} \mathbf{q}}{(2 \pi)^{3}} G_{0}(i \Omega, \mathbf{q}) \sigma_{3} G_{0}(i \Omega, \mathbf{q}) \\
& \times D_{0}(i \Omega, \mathbf{q}) .
\end{aligned}
$$

Substituting Eqs. (A2) and (A4) into Eq. (F52), we arrive at

$$
\delta \lambda_{3}^{(5)}=\lambda_{3} C_{4}^{\prime} \ell
$$

where

$$
\begin{aligned}
C_{4}^{\prime}= & \frac{g^{2}}{12 \pi^{2} \sqrt{A \Lambda}} \int_{0}^{+\infty} d \xi \frac{\xi^{5 / 3}}{\left(1+\xi^{2}\right)^{13 / 12}} \\
& \times \frac{1}{\xi^{2 / 3}\left(1+\xi^{2}\right)^{1 / 6}+\zeta} .
\end{aligned}
$$

Diagrams shown in Figs. 6(f) and 6 (g) lead to

$$
\begin{aligned}
V_{3}^{(6)+(7)}= & -4 g^{2} \lambda_{3} \int^{\prime} \frac{d \Omega}{2 \pi} \frac{d^{3} \mathbf{q}}{(2 \pi)^{3}}\left(\psi_{\sigma}^{\dagger} \sigma_{3} G_{0}(i \Omega, \mathbf{q}) \psi_{\sigma}\right) \\
& \times\left\{\psi^{\dagger}\left[G_{0}(i \Omega, \mathbf{q}) \sigma_{3}+\sigma_{3} G_{0}(-i \Omega,-\mathbf{q})\right] \psi\right\} \\
& \times D_{0}(i \Omega, \mathbf{q}) \\
= & -8 \lambda_{3} g^{2} \int^{\prime} \frac{d \Omega}{2 \pi} \frac{d^{3} \mathbf{q}}{(2 \pi)^{3}} \frac{B^{2} d_{1}^{2}(\mathbf{k})}{\left(\Omega^{2}+E_{\mathbf{k}}^{2}\right)^{2}} D_{0}(i \Omega, \mathbf{q}) \\
& \times\left(\psi^{\dagger} \sigma_{2} \psi\right)^{2} \\
& -8 \lambda_{3} g^{2} \int^{\prime} \frac{d \Omega}{2 \pi} \frac{d^{3} \mathbf{q}}{(2 \pi)^{3}} \frac{B^{2} d_{2}^{2}(\mathbf{k})}{\left(\Omega^{2}+E_{\mathbf{k}}^{2}\right)^{2}} D_{0}(i \Omega, \mathbf{q}) \\
& \times\left(\psi^{\dagger} \sigma_{1} \psi\right)^{2} \\
& -8 \lambda_{3} g^{2} \int^{\prime} \frac{d \Omega}{2 \pi} \frac{d^{3} \mathbf{q}}{(2 \pi)^{3}} \frac{A^{2} d_{3}^{2}(\mathbf{k})}{\left(\Omega^{2}+E_{\mathbf{k}}^{2}\right)^{2}} D_{0}(i \Omega, \mathbf{q}) \\
& \times\left(\psi^{\dagger} \psi\right)^{2} .
\end{aligned}
$$

Using Fierz identity and carrying out the integrations, we obtain

$$
V_{3}^{(6)+(7)}=\lambda_{3} C_{5}^{\prime} \ell\left(\psi^{\dagger} \sigma_{3} \psi\right)^{2}
$$

where

$$
\begin{aligned}
C_{5}^{\prime}= & \frac{g^{2}}{6 \pi^{2} \sqrt{A \Lambda}} \int_{0}^{+\infty} d \xi \frac{1-\xi^{2}}{\xi^{1 / 3}\left(1+\xi^{2}\right)^{13 / 12}} \\
& \times \frac{1}{\xi^{2 / 3}\left(1+\xi^{2}\right)^{1 / 6}+\zeta} .
\end{aligned}
$$

This means that

$$
\delta \lambda_{3}^{(6)+(7)}=\lambda_{3} C_{5}^{\prime} \ell .
$$

The correction for $\lambda_{3}$ from Fig. 6(h) is

$$
\begin{aligned}
\delta \lambda_{3}^{(8)}= & 2 \lambda_{3} g^{2} \int^{\prime} \frac{d \omega}{2 \pi} \frac{d^{3} \mathbf{k}}{(2 \pi)^{3}} \operatorname{Tr}\left[G_{0}(i \omega, \mathbf{k}) \sigma_{3}\right. \\
& \left.\times G_{0}(i \omega+i \Omega, \mathbf{k}+\mathbf{q})\right] D_{0}(i \Omega, \mathbf{q}) \\
= & 0 .
\end{aligned}
$$

Figs. 6(i) and 6(j) yield

$$
\begin{aligned}
V_{3}^{(9)+(10)}= & 4 g^{4} \int^{\prime} \frac{d \Omega}{2 \pi} \frac{d^{3} \mathbf{q}}{(2 \pi)^{3}}\left(\psi^{\dagger} G_{0}(i \Omega, \mathbf{q}) \psi\right) \\
& \times D_{0}(i \Omega, \mathbf{q})\left\{\psi ^ { \dagger } \left[G_{0}(i \Omega, \mathbf{q})\right.\right. \\
& \left.\left.+G_{0}(-i \Omega,-\mathbf{q})\right] \psi\right\} D_{0}(i \Omega, \mathbf{q}) \\
= & \frac{\sqrt{A} B^{2 / 3}}{\Lambda^{1 / 6}} C_{6} \ell\left(\psi^{\dagger} \sigma_{3} \psi\right)^{2},
\end{aligned}
$$

where $C_{6}$ is expressed by Eq. (F37). Thus,

$$
\delta \lambda_{3}^{(9)+(10)}=\frac{\sqrt{A} B^{2 / 3}}{\Lambda^{1 / 6}} C_{6} \ell .
$$

The total correction can be written as

$$
\begin{aligned}
\delta \lambda_{3}= & \left(1-\frac{1}{4 N}\right) \lambda_{3}^{2} \frac{\Gamma\left(\frac{5}{4}\right) \Gamma\left(\frac{4}{3}\right) \Lambda^{1 / 6}}{3 \pi^{2} \Gamma\left(\frac{19}{12}\right) \sqrt{A} B^{2 / 3}} \ell \\
& +\lambda_{3}\left(C_{4}^{\prime}+C_{5}^{\prime}\right) \ell+\frac{\sqrt{A} B^{2 / 3}}{\Lambda^{1 / 6}} C_{6} \ell
\end{aligned}
$$



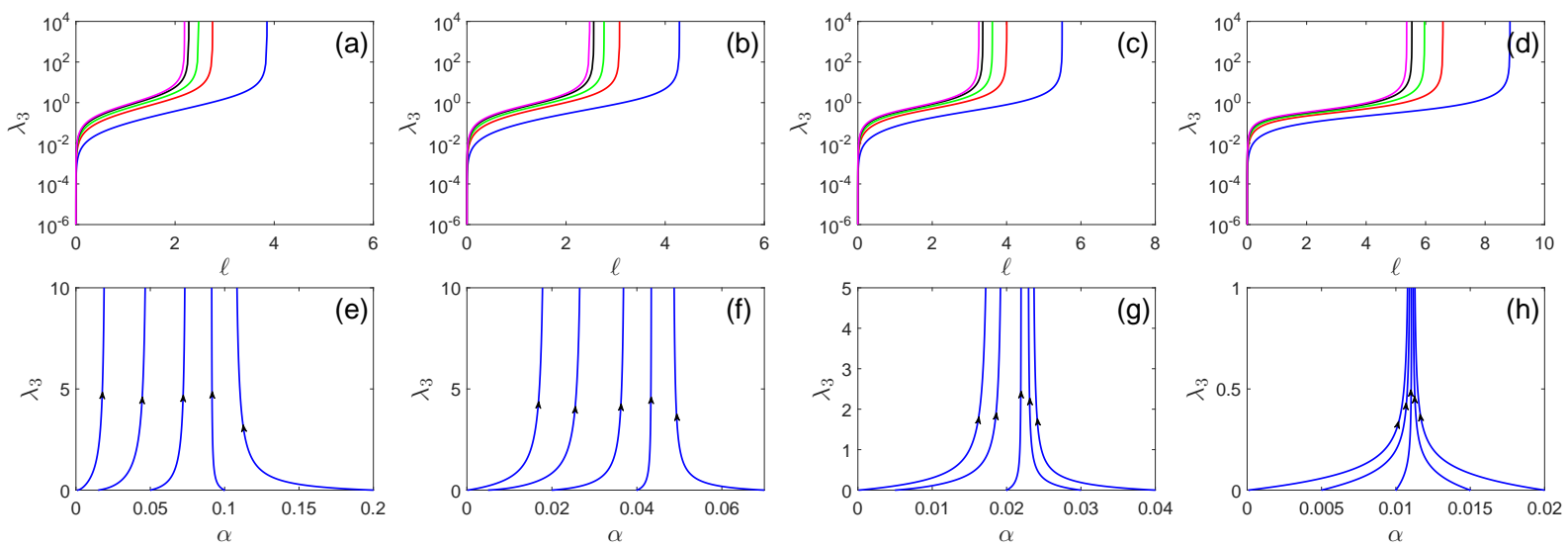

FIG. 8: (a)-(d): Dependence of $\lambda_{3}$ on $\ell$ obtained at $N=1, N=2, N=4, N=8$, respectively. Blue, red, green, black, magenta lines correspond to $\alpha_{0}=0.01,0.05,0.1,0.2,0.3$, respectively. (e)-(h): Flow diagrams on the $\alpha-\lambda_{3}$ plane at $N=1$, $N=2, N=4$, and $N=8$, respectively. Here, we take $\beta_{0}=0.1$.

After incorporating the corrections, the action of fourfermion interaction becomes

$$
\begin{aligned}
S_{\psi^{4}}= & \frac{1}{N}\left(\lambda_{3}+\delta \lambda_{3}\right) \int \frac{d \omega_{1}}{2 \pi} \frac{d^{3} \mathbf{k}_{1}}{(2 \pi)^{3}} \frac{d \omega_{2}}{2 \pi} \frac{d^{3} \mathbf{k}_{2}}{(2 \pi)^{3}} \frac{d \omega_{3}}{2 \pi} \frac{d^{3} \mathbf{k}_{3}}{(2 \pi)^{3}} \\
& \times \psi_{a}^{\dagger}\left(\omega_{1}, \mathbf{k}_{1}\right) \sigma_{3} \psi_{a}\left(\omega_{2}, \mathbf{k}_{2}\right) \psi_{a}^{\dagger}\left(\omega_{3}, k_{3}\right) \sigma_{3} \\
& \times \psi_{a}\left(\omega_{1}-\omega_{2}+\omega_{3}, \mathbf{k}_{1}-\mathbf{k}_{2}+\mathbf{k}_{3}\right) .
\end{aligned}
$$

Utilizing the transformations Eqs. (C3)-(C7), we arrive at

$$
\begin{aligned}
S_{\psi^{\prime 4}} \approx & \frac{1}{N}\left(\lambda_{3}-\lambda_{3} \frac{\ell}{6}+\delta \lambda_{3}\right) \int \frac{d \omega_{1}^{\prime}}{2 \pi} \frac{d^{3} \mathbf{k}_{1}^{\prime}}{(2 \pi)^{3}} \frac{d \omega_{2}^{\prime}}{2 \pi} \frac{d^{3} \mathbf{k}_{2}^{\prime}}{(2 \pi)^{3}} \\
& \times \frac{d \omega_{3}^{\prime}}{2 \pi} \frac{d^{3} \mathbf{k}_{3}^{\prime}}{(2 \pi)^{3}} \psi_{a}^{\prime \dagger}\left(\omega_{1}^{\prime}, \mathbf{k}_{1}^{\prime}\right) \sigma_{3} \psi_{a}^{\prime}\left(\omega_{2}^{\prime}, \mathbf{k}_{2}^{\prime}\right) \psi_{a}^{\prime \dagger}\left(\omega_{3}^{\prime}, k_{3}^{\prime}\right) \\
& \times \sigma_{3} \psi_{a}^{\prime}\left(\omega_{1}^{\prime}-\omega_{2}^{\prime}+\omega_{3}^{\prime}, \mathbf{k}_{1}^{\prime}-\mathbf{k}_{2}^{\prime}+\mathbf{k}_{3}^{\prime}\right) . \quad(\mathrm{F} 64
\end{aligned}
$$

Defining a new parameter

$$
\lambda_{3}^{\prime}=\lambda_{3}-\lambda_{3} \frac{\ell}{6}+\delta \lambda_{3},
$$

we restore the original form of the action. From Eqs. (F62) and (F65), one gets the flow equation for $\lambda_{3}$

$$
\begin{aligned}
\frac{d \lambda_{3}}{d \ell}= & -\frac{1}{6} \lambda_{3}+\frac{4}{5}\left(1-\frac{1}{4 N}\right) \lambda_{3}^{2}+\left(C_{4}^{\prime}+C_{5}^{\prime}-\frac{1}{2} C_{3}\right. \\
& \left.-\frac{2}{3} C_{2}\right) \lambda_{3}+C_{7}
\end{aligned}
$$

where

$$
C_{7}=\frac{5 \Gamma\left(\frac{5}{4}\right) \Gamma\left(\frac{4}{3}\right)}{12 \pi^{2} \Gamma\left(\frac{19}{12}\right)} C_{6}
$$

Once again, we have made the replacement

$$
\frac{5 \Gamma\left(\frac{5}{4}\right) \Gamma\left(\frac{4}{3}\right) \Lambda^{1 / 6}}{12 \pi^{2} \Gamma\left(\frac{19}{12}\right) \sqrt{A} B^{2 / 3}} \lambda_{3} \rightarrow \lambda_{3}
$$

The RG flows of $\lambda_{3}$ for several possible values of fermion flavor $N$ are presented in Figs. 8 (a)- 8 (d). The corresponding flow diagrams on the $\alpha-\lambda_{3}$ plane are shown in Figs. $8(\mathrm{e})-8(\mathrm{~h})$. It is clear that $\lambda_{3}$ grows from zero and becomes divergent at a finite $\ell$. Physically, these results indicate that $\left\langle\psi^{\dagger} \sigma_{3} \psi\right\rangle \neq 0$ due to the Coulomb interaction. According to the above analysis, the TQPT between triple-WSM and BI is continuous if $\left\langle\psi^{\dagger} \sigma_{3} \psi\right\rangle=0$, but is first order once $\left\langle\psi^{\dagger} \sigma_{3} \psi\right\rangle$ becomes finite.

We now know that the system could either enter into a gapless nematic phase or undergo a first order TQPT. It is necessary to determine which instability is more favorable at low energies. For this purpose, we now compare the RG flows of the coupling parameters $\lambda_{1}$ and $\lambda_{3}$. According to the results shown in Fig. 9 we find that the Coulomb interaction, within a wide range of initial values of $\alpha$, drives $\lambda_{3}$ to diverge more quickly as the energy decreases for fermion flavors $N=1$ and $N=2$. However, $\lambda_{1}$ becomes divergent more quickly for flavors $N \geq 3$. We conclude that the Coulomb interaction tends to trigger first order TQPT for $N=1,2$, but leads to a gapless nematic state for $N \geq 3$.

\section{Interplay with $\left(\psi^{\dagger} \psi\right)^{2}$}

The correction induced by Fig. 6(a) satisfies

$$
\begin{aligned}
\delta \lambda_{0}^{(1)} & =\frac{4 \lambda_{0}^{2}}{N} \int^{\prime} \frac{d \omega}{2 \pi} \frac{d^{3} \mathbf{k}}{(2 \pi)^{3}} G_{0}(i \omega, \mathbf{k}) G_{0}(i \omega, \mathbf{k}) \\
& =0
\end{aligned}
$$

The correction resulting from Figs. 6(b) and 6(c) takes the form

$$
\begin{aligned}
V_{0}^{(2)+(3)}= & \frac{4 \lambda_{0}^{2}}{N} \int^{\prime} \frac{d \omega}{2 \pi} \frac{d^{3} \mathbf{k}}{(2 \pi)^{3}}\left(\psi^{\dagger} G_{0}(i \omega, \mathbf{k}) \psi\right) \\
& \times\left\{\psi^{\dagger}\left[G_{0}(i \omega, \mathbf{k})+G_{0}(-i \omega,-\mathbf{k})\right] \psi\right\}
\end{aligned}
$$



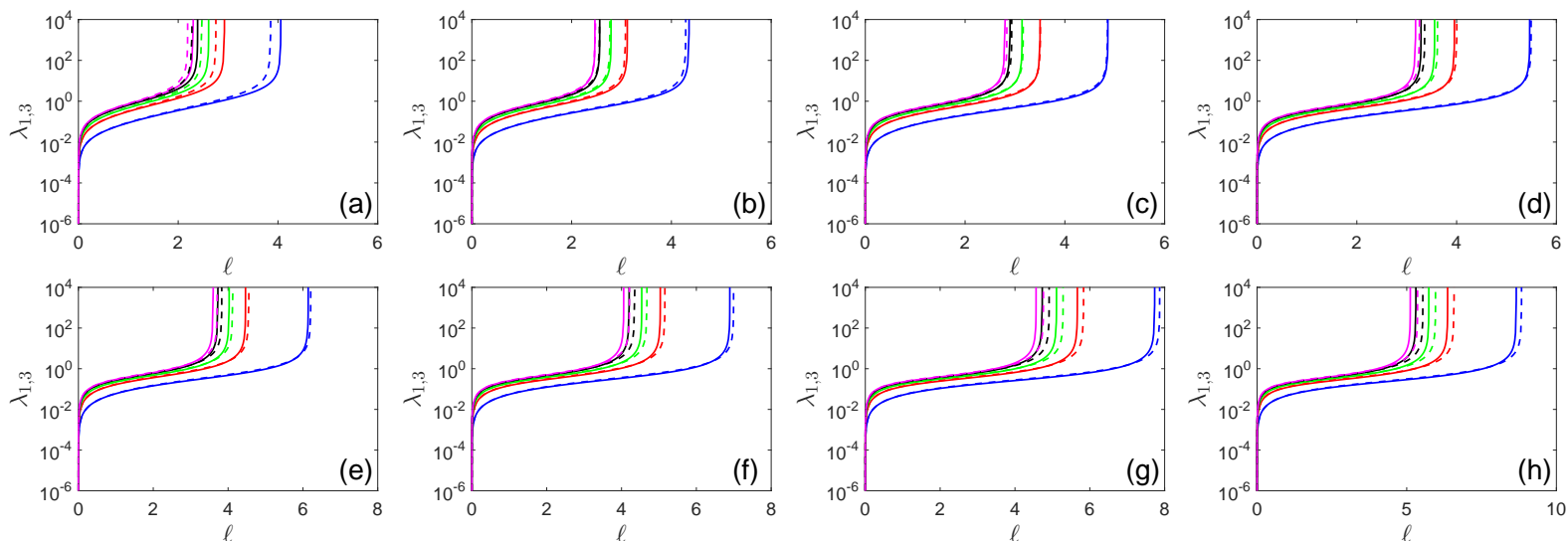

FIG. 9: Solid line corresponds to the flow of $\lambda_{1}$. Dashed line represents the flow of $\lambda_{3}$. Blue, red, green, black, and magenta lines correspond to $\alpha_{0}=0.01,0.05,0.1,0.2$, and 0.3 respectively. In (a)-(h), the flavor is $N=1,2,3,4,5,6,7,8$ respectively. Here, $\beta_{0}=0.1$.
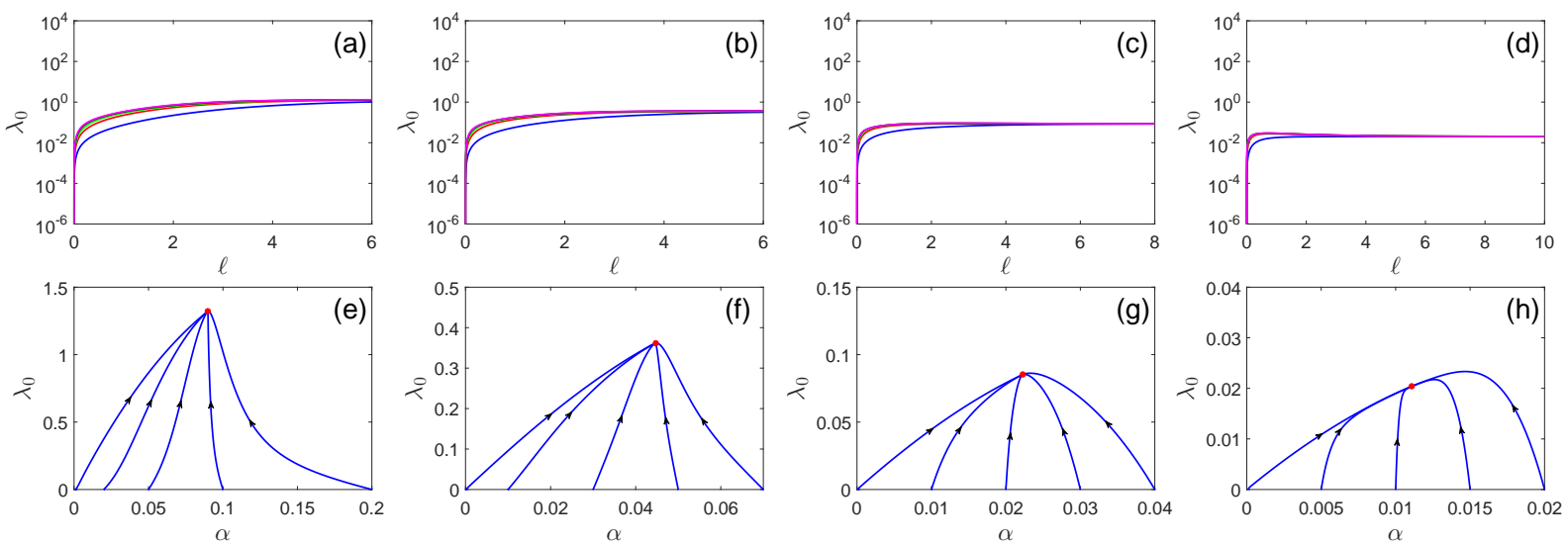

FIG. 10: (a)-(d): Dependence of $\lambda_{0}$ on $\ell$ with $N=1, N=2, N=4, N=8$ respectively. Blue, red, green, black, and magenta lines correspond to $\alpha_{0}=0.01,0.05,0.1,0.2$, and 0.3 respectively. (e)-(h): Flow diagrams on the plane of the parameters $\alpha$ and $\lambda_{0}$ with $N=1, N=2, N=4$, and $N=8$ respectively. Here, we take $\beta_{0}=0.1$.

$$
=\frac{\lambda_{0}^{2}}{N} \frac{\Gamma\left(\frac{1}{3}\right) \Gamma\left(\frac{5}{4}\right) \Lambda^{1 / 6}}{12 \pi^{2} \Gamma\left(\frac{19}{12}\right) \sqrt{A} B^{2 / 3}} \ell\left(\psi^{\dagger} \sigma_{3} \psi\right)^{2}(\mathrm{~F} 70)
$$

which is equivalent to

$$
V_{0}^{(2)+(3)}=-\frac{\lambda_{0}^{2}}{N} \frac{\Gamma\left(\frac{1}{3}\right) \Gamma\left(\frac{5}{4}\right) \Lambda^{1 / 6}}{12 \pi^{2} \Gamma\left(\frac{19}{12}\right) \sqrt{A} B^{2 / 3}} \ell\left(\psi^{\dagger} \psi\right)^{2},
$$

according to Fierz identity. Thus $\delta \lambda_{0}^{(2)+(3)}$ can be written as

$$
\delta \lambda_{0}^{(2)+(3)}=-\frac{\lambda_{0}^{2}}{N} \frac{\Gamma\left(\frac{1}{3}\right) \Gamma\left(\frac{5}{4}\right) \Lambda^{1 / 6}}{12 \pi^{2} \Gamma\left(\frac{19}{12}\right) \sqrt{A} B^{2 / 3}} \ell .
$$

Diagram shown in Fig. [6(d) generates the correction

$$
\begin{aligned}
\delta \lambda_{0}^{(4)} & =-2 N \frac{\lambda_{0}^{2}}{N} \int^{\prime} \frac{d \omega}{2 \pi} \frac{d^{3} \mathbf{k}}{(2 \pi)^{3}} \operatorname{Tr}\left[G_{0}(i \omega, \mathbf{k}) G_{0}(i \omega, \mathbf{k})\right] \\
& =0 .
\end{aligned}
$$

The correction due to Fig. 6(e) reads

$$
\begin{aligned}
\delta \lambda_{0}^{(5)}= & -2 g^{2} \lambda_{0} \int^{\prime} \frac{d \Omega}{2 \pi} \frac{d^{3} \mathbf{q}}{(2 \pi)^{3}} G_{0}(i \Omega, \mathbf{q}) G_{0}(i \Omega, \mathbf{q}) \\
& \times D_{0}(i \Omega, \mathbf{q}) \\
= & 0 .
\end{aligned}
$$

Figs. 6(f) and 6(g) yield

$$
\begin{aligned}
V_{0}^{(6)+(7)}= & -4 g^{2} \lambda_{0} \int^{\prime} \frac{d \Omega}{2 \pi} \frac{d^{3} \mathbf{q}}{(2 \pi)^{3}}\left(\psi_{\sigma}^{\dagger} G_{0}(i \Omega, \mathbf{q}) \psi_{\sigma}\right) \\
& \times\left\{\psi^{\dagger}\left[G_{0}(i \Omega, \mathbf{q})+G_{0}(-i \Omega,-\mathbf{q})\right] \psi\right\} \\
& \times D_{0}(i \Omega, \mathbf{q}) \\
= & -\lambda_{0} C_{5}^{\prime \prime} \ell\left(\psi^{\dagger} \sigma_{3} \psi\right)^{2} .
\end{aligned}
$$

Using Fierz identity, we re-express $V^{(6)+(7)}$ as

$$
V_{0}^{(6)+(7)}=\lambda_{0} C_{5}^{\prime \prime} \ell\left(\psi^{\dagger} \psi\right)^{2}
$$


where

$$
\begin{aligned}
C_{5}^{\prime \prime}= & \frac{g^{2}}{6 \pi^{2} \sqrt{A \Lambda}} \int_{0}^{+\infty} d \xi \frac{1}{\xi^{1 / 3}\left(1+\xi^{2}\right)^{13 / 12}} \\
& \times \frac{1}{\xi^{2 / 3}\left(1+\xi^{2}\right)^{1 / 6}+\zeta} .
\end{aligned}
$$

Thus, we obtain

$$
\delta \lambda_{0}^{(6)+(7)}=\lambda_{0} C_{5}^{\prime \prime} \ell
$$

Fig. 6(h) results in

$$
\begin{aligned}
\delta \lambda_{0}^{(8)}= & 2 \lambda_{0} N g^{2} \int^{\prime} \frac{d \omega}{2 \pi} \frac{d^{3} \mathbf{k}}{(2 \pi)^{3}} \operatorname{Tr}\left[G_{0}(i \omega, \mathbf{k})\right. \\
& \left.\times G_{0}(i \omega+i \Omega, \mathbf{k}+\mathbf{q})\right] D_{0}(i \Omega, \mathbf{q}) \\
\approx & -2 \lambda_{0} N\left(C_{\perp}+\frac{C_{z}}{\eta}\right) \ell .
\end{aligned}
$$

The corrections induced by Figs. 6(i) and 6(j) are

$$
\begin{aligned}
V_{0}^{(9)+(10)}= & 4 g^{4} \int^{\prime} \frac{d \Omega}{2 \pi} \frac{d^{3} \mathbf{q}}{(2 \pi)^{3}}\left(\psi^{\dagger} G_{0}(i \Omega, \mathbf{q}) \psi\right) \\
& \times D_{0}(i \Omega, \mathbf{q})\left\{\psi ^ { \dagger } \left[G_{0}(i \Omega, \mathbf{q})\right.\right. \\
& \left.\left.+G_{0}(-i \Omega,-\mathbf{q})\right] \psi\right\} D_{0}(i \Omega, \mathbf{q}) \\
\approx & \frac{\sqrt{A} B^{2 / 3}}{\Lambda^{1 / 6}} C_{6} \ell\left(\psi^{\dagger} \sigma_{3} \psi\right)^{2}
\end{aligned}
$$

which can be further written as

$$
V_{0}^{(9)+(10)}=-\frac{\sqrt{A} B^{2 / 3}}{\Lambda^{1 / 6}} C_{6} \ell\left(\psi^{\dagger} \psi\right)^{2} .
$$

Accordingly, we find

$$
\delta \lambda_{0}^{(9)+(10)}=-\frac{\sqrt{A} B^{2 / 3}}{\Lambda^{1 / 6}} C_{6} \ell,
$$

where $C_{6}$ is given by Eq. (F37).

The total correction to $\lambda_{0}$ is

$$
\begin{aligned}
\delta \lambda_{0}= & -\frac{\lambda_{0}^{2}}{N} \frac{\Gamma\left(\frac{1}{3}\right) \Gamma\left(\frac{5}{4}\right) \Lambda^{1 / 6}}{12 \pi^{2} \Gamma\left(\frac{19}{12}\right) \sqrt{A} B^{2 / 3}} \ell+\lambda_{0}\left(C_{5}^{\prime \prime}-2 N C_{\perp}\right. \\
& \left.-2 N \frac{C_{z}}{\eta}\right) \ell-\frac{\sqrt{A} B^{2 / 3}}{\Lambda^{1 / 6}} C_{6} \ell
\end{aligned}
$$

We then obtain the following RG equation for $\lambda_{0}$

$$
\begin{aligned}
\frac{d \lambda_{0}}{d \ell}= & -\frac{1}{6} \lambda_{0}-\frac{3}{5 N} \lambda_{0}^{2}+\left(C_{5}^{\prime \prime}-2 N C_{\perp}-2 N \beta\right) \lambda_{0} \\
& -C_{7},
\end{aligned}
$$

where

$$
C_{7}=\frac{5 \Gamma\left(\frac{5}{4}\right) \Gamma\left(\frac{4}{3}\right)}{12 \pi^{2} \Gamma\left(\frac{19}{12}\right)} C_{6} .
$$

The following re-definition

$$
\frac{5 \Gamma\left(\frac{4}{3}\right) \Gamma\left(\frac{5}{4}\right) \Lambda^{1 / 6}}{12 \pi^{2} \Gamma\left(\frac{19}{12}\right) \sqrt{A} B^{2 / 3}} \lambda_{0} \rightarrow \lambda_{0}
$$

has been introduced during the derivation of RG equation.

The $\ell$-dependence of $\lambda_{0}$ can be seen from Figs. 10(a)10(d). Different from $\lambda_{1}$ and $\lambda_{3}, \lambda_{0}$ flows to certain constant as $\ell \rightarrow \infty$. The flow diagrams on the $\alpha-\lambda_{0}$ are depicted in Figs. 10(e)-10(h). Apparently, in this case the interplay between Coulomb and the four-fermion interaction does not lead to any instability of the system. The expectation value $\left\langle\psi^{\dagger} \psi\right\rangle$ always vanishes.
[1] G. F. Giuliani and G. Vignale, Quantum Theory of the Electron Liquid (Cambridge University Press, Cambridge, 2005).

[2] P. Coleman, Introduction to Many-Body Physics (Cambridge University Press, Cambridge, 2015).

[3] S. Sachdev, Quantum Phase Transitions (Cambridge University Press, Cambridge, 2011).

[4] H. v. Löhneysen, A. Rosch, M. Vojta, and P. Wölfle, Fermi-liquid instabilities at magnetic quantum phase transitions, Rev. Mod. Phys. 79, 1015 (2007).

[5] E. Fradkin, S. A. Kivelson, and J. M. Tranquada, Theory of intertwined orders in high temperature superconductors, Rev. Mod. Phys. 87, 457 (2015).

[6] E. Fradkin, S. A. Kivelson, M. J. Lawler, J. P. Eisenstein, and A. P. Mackenzie, Nematic Fermi fluids in condensed matter physics, Annu. Rev. Condens. Matter Phys. 1, 153 (2010).

[7] C. M. Varma, Z. Nussinov, and W. v. Saarloos, Singular or non-Fermi liquids, Phys. Rep. 361, 267 (2002).

[8] X.-G. Wen, Quantum Field Theory of Many-Body Systems (Oxford University Press, Oxford, 2004).

[9] X.-G. Wen, Zoo of quantum-topological phases of matter, Rev. Mod. Phys. 89, 041004 (2017).

[10] V. N. Kotov, B. Uchoa, V. M. Pereira, F. Guinea, and A. H. Castro Neto, Electron-electron interactions in graphene: Current status and perspectives, Rev. Mod. Phys. 84, 1067 (2012).

[11] O. Vafek and A. Vishwanath, Dirac fermions in solids: From high- $\mathrm{T}_{c}$ cuprates and graphene to topological insulators and Weyl semimetals, Annu. Rev. Condens. Matter Phys. 5, 83 (2014).

[12] T. O. Wehling, A. M. Black-Schaffer, and A. V. Balatsky, Dirac materials, Adv. Phys. 63, 1 (2014).

[13] X. Wan, A. M. Turner, A. Vishwanath, and S. Y. Savrasov, Topological semimetal and Fermi-arc surface states in the electronic structure of pyrochlore iridates, 
Phys. Rev. B 83, 205101 (2011).

[14] H. Weng, X. Dai, and Z. Fang, Topological semimetals predicted from first-principles calculations, J. Phys.: Condens. Matter 28, 303001 (2016).

[15] C. Fang, H. Weng, X. Dai, and Z. Fang, Topological nodal line semimetals, Chin. Phys. B 25, 117106 (2016).

[16] B. Yan and C. Felser, Topological materials: Weyl semimetals, Annu. Rev. Condens. Matter Phys. 8, 337 (2017).

[17] M. Z. Hasan, S.-Y. Xu, I. Belopolski, and S.-M. Huang, Discovery of Weyl fermion semimetals and topological Fermi arc states, Annu. Rev. Condens. Matter Phys. 8, 289 (2017).

[18] N. P. Armitage, E. J. Mele, and A. Vishwanath, Weyl and Dirac semimetals in three-dimensional solids, Rev. Mod. Phys. 90, 015001 (2018).

[19] D. T. Son and B. Z. Spivak, Chiral anomaly and classical negative magnetoresistance of Weyl metals, Phys. Rev. B 88, 104412 (2013); A. A. Burkov, Chiral anomaly and diffusive magnetotransport in Weyl metals, Phys. Rev. Lett. 113, 247203 (2014).

[20] A. A. Burkov, Giant planar Hall effect in topological metals, Phys. Rev. B 96, 041110(R) (2017); S. Nandy, G. Sharma, A. Taraphder, and S. Tewari, Chiral anomaly as the origin of the planar Hall effect in Weyl semimetals, Phys. Rev. Lett. 119, 176804 (2017).

[21] S. Murakami, Phase transition between the quantum spin Hall and insulator phases in 3D: Emergence of a topological gapless, New J. Phys. 9, 356 (2007).

[22] G. Montambaux, F. Piéchon, J.-N. Fuchs, and M. O. Goerbig, Merging of Dirac points in a two-dimensional crystal, Phys. Rev. B 80, 153412 (2009).

[23] P. Goswami and S. Chakravarty, Quantum criticality between topological and band insulators in $3+1$ dimensions, Phys. Rev. Lett. 107, 196803 (2011).

[24] H. Isobe, B.-J. Yang, A. Chubukov, J. Schmalian, and N. Nagaosa, Emergent non-Fermi-liquid at the quantum critical point of a topological phase transition in two dimensions, Phys. Rev. Lett. 116, 076803 (2016).

[25] G. Y. Cho and E.-G. Moon, Novel quantum criticality in two dimensional topological phase transitions, Sci. Rep. 6, 19198 (2016).

[26] J.-R. Wang, G.-Z. Liu, and C.-J. Zhang, Excitonic pairing and insulating transition in two-dimensional semiDirac semimetals, Phys. Rev. B 95, 075129 (2017).

[27] J. Ahn and B.-J. Yang, Unconventional topological phase transition in two-dimensional systems with space-time inversion symmetry, Phys. Rev. Lett. 118, 156401 (2017).

[28] B. Roy and M. S. Foster, Quantum multicriticality near the Dirac-semimetal to band-insulator critical point in two dimensions: a controlled ascent from one dimension, Phys. Rev. X 8, 011049 (2018).

[29] B.-J. Yang, M. S. Bahramy, R. Arita, H. Isobe, E.-G. Moon, and N. Nagaosa, Theory of topological quantum phase transitions in 3D noncentrosymmetric systems, Phys. Rev. Lett. 110, 086402 (2013).

[30] B.-J. Yang and N. Nagaosa, Classification of stable threedimensional Dirac semimetals with nontrivial topology, Nat. Commun. 5, 4898 (2014).

[31] B.-J. Yang, E.-G. Moon, H. Isobe, and N. Nagaosa, Quantum criticality of topological phase transitions in three-dimensional interacting electronic systems, Nat. Phys. 10, 774 (2014).

[32] S. Murakami, M. Hirayama, R. Okugawa, and T. Miyake,
Emergence of topological semimetals in gap closing in semiconductors without inversion symmetry, Sci. Adv. 3, e1602680 (2017).

[33] B. Roy, R.-J. Slager, and V. Juričić, Global phase diagram of a dirty Weyl liquid and emergent superuniversality, Phys. Rev. X 8, 031076 (2018).

[34] S.-Y. Xu, Y. Xia, L. A. Wray, S. Jia, F. Meier, J. H. Dil, J. Osterwalder, B. Slomski, A. Bansil, H. Lin, R. J. Cava, and M. Z. Hasan, Topological phase transition and texture inversion in a tunable topological insulator, Science 332, 560 (2011).

[35] T. Sato, K. Segawa, K. Kosaka, S. Souma, K. Nakayama, K. Eto, T. Minami, Y. Ando, and T. Takahashi, Unexpected mass acquisition of Dirac fermions at the quantum phase transition of a topological insulator, Nat. Phys. 7, 840 (2011).

[36] E.-G. Moon, C. Xu, Y. B. Kim, and L. Balents, NonFermi-liquid and topological states with strong spin-orbit coupling, Phys. Rev. Lett. 111, 206401 (2013).

[37] I. F. Herbut and L. Janssen, Topological Mott insulator in three-dimensional systems with quadratic band touching, Phys. Rev. Lett. 113, 106401 (2014).

[38] L. Janssen and I. F. Herbut, Phase diagram of electronic systems with quadratic Fermi nodes in $2<d<4$ : $2+\epsilon$ expansion, $4-\epsilon$ expansion, and functional renormalization group, Phys. Rev. B 95, 075101 (2017).

[39] H.-H. Lai, Correlation effects in double-Weyl semimetals, Phys. Rev. B 91, 235131 (2015).

[40] S.-K. Jian and H. Yao, Correlated double-Weyl semimetals with Coulomb interactions: Possible applications to $\mathrm{HgCr}_{2} \mathrm{Se}_{4}$ and $\mathrm{SrSi}_{2}$, Phys. Rev. B 92, 045121 (2015).

[41] Y. Huh, E.-G. Moon, and Y. B. Kim, Long-range Coulomb interaction in nodal-ring semimetals, Phys. Rev. B 93, 035138 (2016).

[42] J.-R. Wang, G.-Z. Liu, and C.-J. Zhang, Quantum phase transition and unusual critical behavior in multi-Weyl semimetals, Phys. Rev. B 96, 165142 (2017).

[43] S.-X. Zhang, S.-K. Jian, and H. Yao, Correlated tripleWeyl semimetals with Coulomb interactions, Phys. Rev. B 96, 241111(R) (2017).

[44] J.-R. Wang, G.-Z. Liu, and C.-J. Zhang, Breakdown of Fermi liquid theory in topological multi-Weyl semimetals, Phys. Rev. B 98, 205113 (2018).

[45] G. Xu, H. Weng, Z. Wang, X. Dai, and Z. Fang, Chern semimetal and the quantized anomalous Hall effect in $\mathrm{HgCr}_{2} \mathrm{Se}_{4}$, Phys. Rev. Lett. 107, 186806 (2011).

[46] C. Fang, M. J. Gilbert, X. Dai, and B. A. Bernevig, Multi-Weyl topological semimetals stabilized by point group symmetry, Phys. Rev. Lett. 108, 266802 (2012).

[47] S. Ahn, E. J. Mele, and H. Min, Optical conductivity of multi-Weyl semimetals, Phys. Rev. B 95, 161112(R) (2017).

[48] S. Park, S. Woo, E. J. Mele, and H. Min, Semiclassical Boltzmann transport theory for multi-Weyl semimetals, Phys. Rev. B 95, 161113(R) (2017).

[49] B. Roy, P. Goswami, and V. Juričić, Interacting Weyl fermions: Phases, phase transitions, and global phase diagram, Phys. Rev. B 95, 201102(R) (2017).

[50] Z.-M. Huang, J. Zhou, and S.-Q. Shen, Topological responses from chiral anomaly in multi-Weyl semimetals, Phys. Rev. B 96, 085201 (2017).

[51] T. Hayata, Y. Kikuchi, and Y. Tanizaki, Topological properties of the chiral magnetic effect in multi-Weyl semimetals, Phys. Rev. B 96, 085112 (2017). 
[52] E. V. Gorbar, V. A. Miransky, I. A. Shovkovy, and P. O. Sukhachov, Anomalous thermoelectric phenomena in lattice models of multi-Weyl semimetals, Phys. Rev. B 96, 155138 (2017).

[53] M. Ezawa, Merging of momentum-space monopoles by controlling Zeeman field: From cubic-Dirac to tripleWeyl fermion systems, Phys. Rev. B 96, 161202(R) (2017).

[54] R. M. A. Dantas, F. Peña-Benitez, B. Roy, and P. Surówka, Magnetotransport in multi-Weyl semimetals: a kinetic theory approach, J. High Energy Phys. 12, 69 (2018).

[55] R. Shankar, Renormalization-group approach to interacting fermions, Rev. Mod. Phys. 66, 129 (1994).

[56] V. Juričić, I. F. Herbut, and G. W. Semenoff, Coulomb interaction at the metal-insulator critical point in graphene, Phys. Rev. B 80, 081405(R) (2009).

[57] B. Roy and S. Das Sarma, Quantum phases of interacting electrons in three-dimensional dirty Dirac semimetals, Phys. Rev. B 94, 115137 (2016).

[58] Q. Liu and A. Zunger, Predicted realization of cubic Dirac fermion in quasi-one-dimensional transition-metal monochalcogenides, Phys. Rev. X 7, 021019 (2017).

[59] W. C. Yu, X. Zhou, F.-C. Chuang, S. A. Yang, H. Lin, and A. Bansil, Nonsymmorphic cubic Dirac point and crossed nodal rings across the ferroelectric phase transition in $\mathrm{LiOsO}_{3}$, Phys. Rev. Materials. 2, 051201(R) (2018).

[60] J. E. Drut and T. A. Lähde, Is graphene in vacuum an insulator?, Phys. Rev. Lett. 102, 026802 (2009).

[61] M. V. Ulybyshev, P. V. Buividovich, M. I. Katsnelson, and M. I. Polikarpov, Monte Carlo study of the semimetal-insulator phase transition in monolayer graphene with a realistic interelectron interaction potential, Phys. Rev. Lett. 111, 056801 (2013).

[62] I. S. Tupitsyn and N. V. Prokof'ev, Stability of Dirac liquids with strong coulomb interaction, Phys. Rev. Lett. 118, 026403 (2017).

[63] H.-K. Tang, J. N. Leaw, J. N. B. Rodrigues, I. F. Herbut, P. Sengupta, F. F. Assaad, and S. Adam, The role of electron-electron interactions in two-dimensional Dirac fermions, Science 361, 570 (2018).

[64] S. Han, C. Lee, E-G. Moon, and H. Min, Emergent anisotropic non-Fermi liquid, arXiv:1809.10691

[65] S.-X. Zhang, S.-K. Jian, and H. Yao, Quantum criticality preempted by nematicity, arXiv:1809.10686 